\title{
Revealing the fate of the phenylcoumaran linkage during lignin oxidation reactions
}

Ciaran W. Lahive ${ }^{\dagger \ddagger}$, Christopher S. Lancefield ${ }^{+\neq *}$, Anna Codina, Paul C. J. Kamer ${ }^{\dagger, \#}$ and Nicholas J. Westwood ${ }^{+}, *$

'School of Chemistry and Biomedical Sciences Research Complex, University of St Andrews and EaStCHEM, Purdie Building, North Haugh, St Andrews, Fife KY16 9ST, United Kingdom

§Bruker UK Ltd., Banner Lane, Coventry, CV4 9GH, United Kingdom

\#Leibniz-Institut für Katalyse e.V., Albert-Einstein-Straße 29a, 18059 Rostock, Germany

₹These authors contributed equally

*E-mail: njw3@st-andrews.ac.uk or c.s.lancefield@uu.nl

Contents

S1.0 General remarks .2

S2.0 General procedure for screening model compounds reactivity with DDQ ...............................

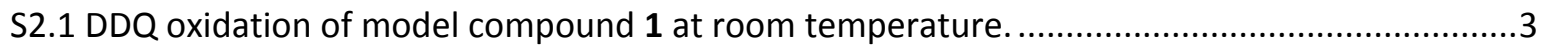

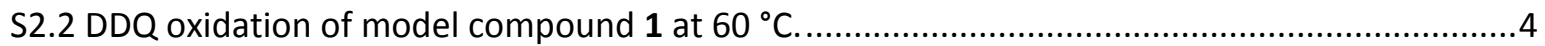

S2.3 DDQ oxidation of model compound $\mathbf{2}$ at room temperature...............................................

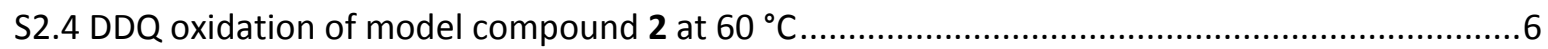

S2.5 DDQ oxidation of model compound $\mathbf{3}$ at room temperature...............................................

S2.6 DDQ oxidation of model compound 4 at room temperature ................................................

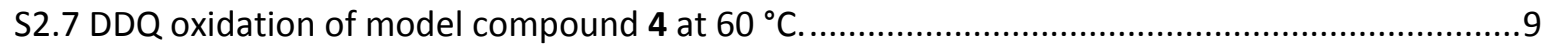

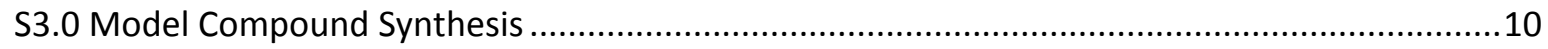

S4.0 Upscaled DDQ oxidations for product isolation and charachterisation...................................10

S5.0 Oxidation of model softwood lignin polymer. .......................................................................13

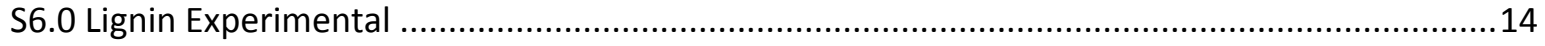

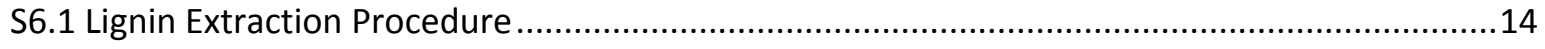

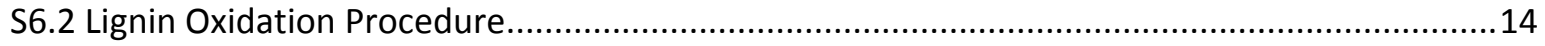

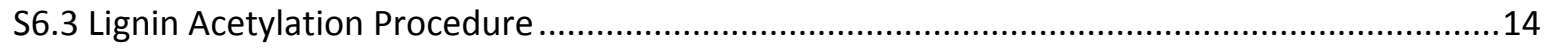

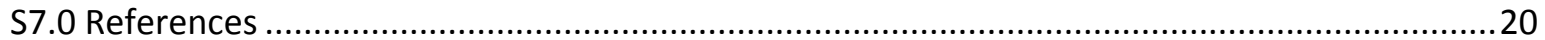

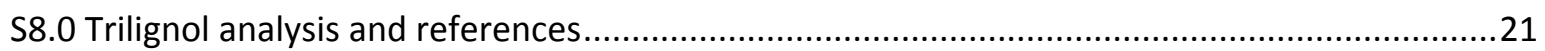

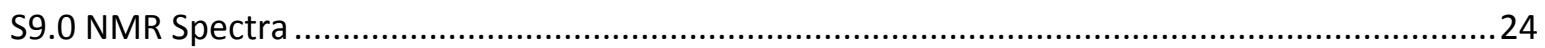




\section{$\underline{\text { S1.0 General remarks }}$}

Commercially available compounds were purchased and used as received unless otherwise stated. DDQ was recrystalised from chloroform prior to use.

NMR: ${ }^{1} \mathrm{H}$ and ${ }^{13} \mathrm{C}$ NMR analysis was performed on a Bruker Avance II $400 \mathrm{MHz}$, Bruker Avance 500 $\mathrm{MHz}$ or a Bruker Avance III $500 \mathrm{MHz}$ spectrometer with the residual solvent peak used as the chemical shift $(\delta)$ reference. Multiplicities are provided using the following abbreviations: $\mathrm{s}=$ singlet, $\mathrm{d}=$ doublet, $\mathrm{t}=$ triplet, $\mathrm{q}=$ quartet and $\mathrm{m}=$ multiplet and the $\mathrm{J}$ couplings are reported in Hz. NMR spectra were processed using TopSpin 3.5 or MestReNova 11.0.

Lignin NMR analysis was performed on Bruker Avance III $500 \mathrm{MHz}$ or $700 \mathrm{MHz}$ spectrometers equipped with nitrogen cooled cryoprobes (Prodigy) using previously described methods as follows: ${ }^{1}$ The ${ }^{1} \mathrm{H}-{ }^{13} \mathrm{C}-\mathrm{HSQC}$ experiment was acquired using standard Bruker pulse sequence 'hsqcetgpsp.2' (phase-sensitive gradient-edited-2D HSQC using adiabatic pulses for inversion and refocusing). Composite pulse sequence 'garp4' was used for broadband decoupling during acquisition. In general 2048 data points was acquired over $12 \mathrm{ppm}$ spectral width (acquisition time $170 \mathrm{~ms}$ ) in F2 dimension using 24 scans with $1 \mathrm{~s}$ interscan delay and the $\mathrm{d} 4$ delay was set to $1.8 \mathrm{~ms}(1 / 4 \mathrm{~J}, \mathrm{~J}=140 \mathrm{~Hz})$. A spectral width of $86 \mathrm{ppm}(47-133 \mathrm{ppm})$ and 128 increments were acquired in F1 dimension (acquisition time $5.9 \mathrm{~ms})$. The spectrum was processed using squared cosinebell in both dimensions and LPfc linear prediction (32 coefficients) in F1. $d_{6}$-DMSO was used as the solvent for unacetylated lignins and $\mathrm{CDCl}_{3}$ for acetylated lignin.

Column chromatography was performed using Fluorochem Silicagel (60§ 40-63 micron).

Thin layer chromatography was performed on pre-coated aluminium plates (60/kieselguhr $\mathrm{F}_{254}$ Merk) and visualized under UV light $\left(254 \mathrm{~nm}\right.$ ) or by staining with $\mathrm{KMnO}_{4}, 1 \%$ iron (III) chloride (for phenolics) or 2,4-dinitrophenylhydrazine (for aldehydes and ketones) where appropriate.

Mass spectrometry data were acquired through the University of St Andrews School of Chemistry mass spectrometry service or through the EPSRC national mass spectrometry service center (Swansea, UK).

IR spectra were obtained on a Shimadzu IRAffinity-1S FourierTransform IR spectrophotometer as thin films. Analysis was carried out using Shimadzu IRsolution v1.50 and only characteristic peaks are reported.

Melting points were recorded on a Gallenkamp melting point apparatus. 
$\underline{\text { S2.0 General procedure for screening model compounds reactivity with DDQ }}$

Substrate (e.g. Compound $1,10 \mathrm{mg}, 0.028 \mathrm{mmol}$ ) was placed in a $3 \mathrm{~mL}$ screw cap vial equipped with a magnetic stirring bar. DDQ (e.g. $6.3 \mathrm{mg}, 0.028 \mathrm{mmol}$ ) was added to the vial followed by 1,4-dioxane $(0.84 \mathrm{~mL})$. The reaction was allowed to stir at either room temperature or at $60{ }^{\circ} \mathrm{C}$ (in an oil bath) for 24 hours. The reaction was then filtered through a cotton wool plug, aq. $\mathrm{NaHCO}_{3}$ was added and the product extracted in to DCM (x3). The combined organic extracts were washed several times with $\mathrm{H}_{2} \mathrm{O}$, brine, dried with $\mathrm{MgSO}_{4}$ and concentrated in vacuo. The concentrated product was then placed in a desiccator under vacuum overnight prior to being analysed by ${ }^{1} \mathrm{H}$ NMR. See Figure S1-Figure $\mathbf{S 7}$ for crude NMR analyses.

S2.1 DDQ oxidation of model compound 1 at room temperature.<smiles>[Y3][R10]([H])(C)[R10](C)(C)C</smiles>

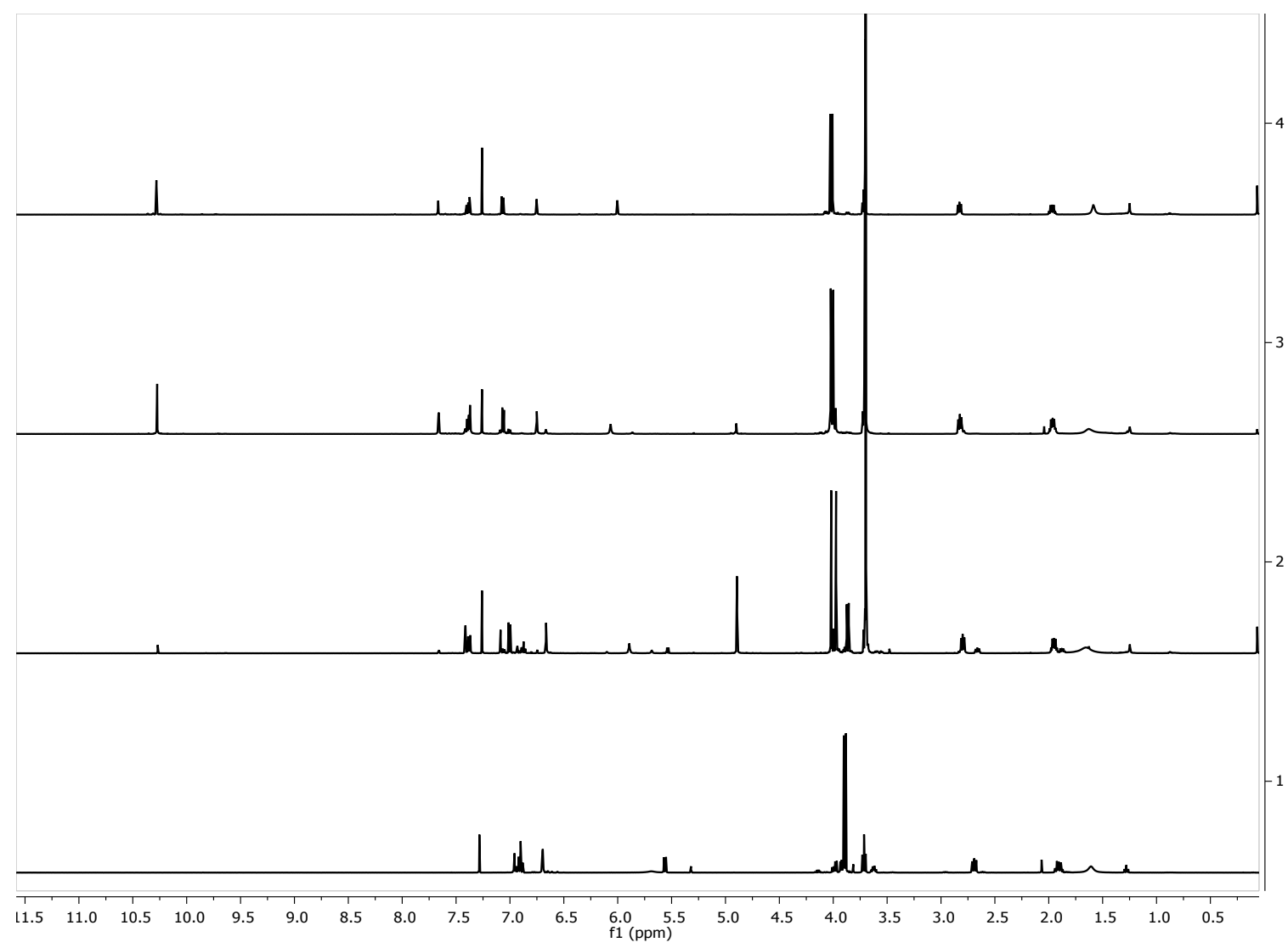

Figure S1: Crude ${ }^{1} \mathrm{H}$ NMR spectra obtained following the reaction of phenolic $\beta-5$ model compound (1) with X equivalents of DDQ at room temperature in 1,4-dioxane. 1) Starting material. 2) 1 eq. 3) 2 eq. and 4) 3 eq. 


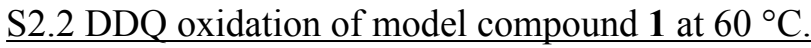<smiles>COc1cc(C2Oc3c(OC)cc(CCCO)cc3[C@@H]2CO)ccc1O</smiles>

1<smiles>COc1cc(-c2oc3c(OC)cc(/C=C/C=O)cc3c2C=O)ccc1O</smiles>

7

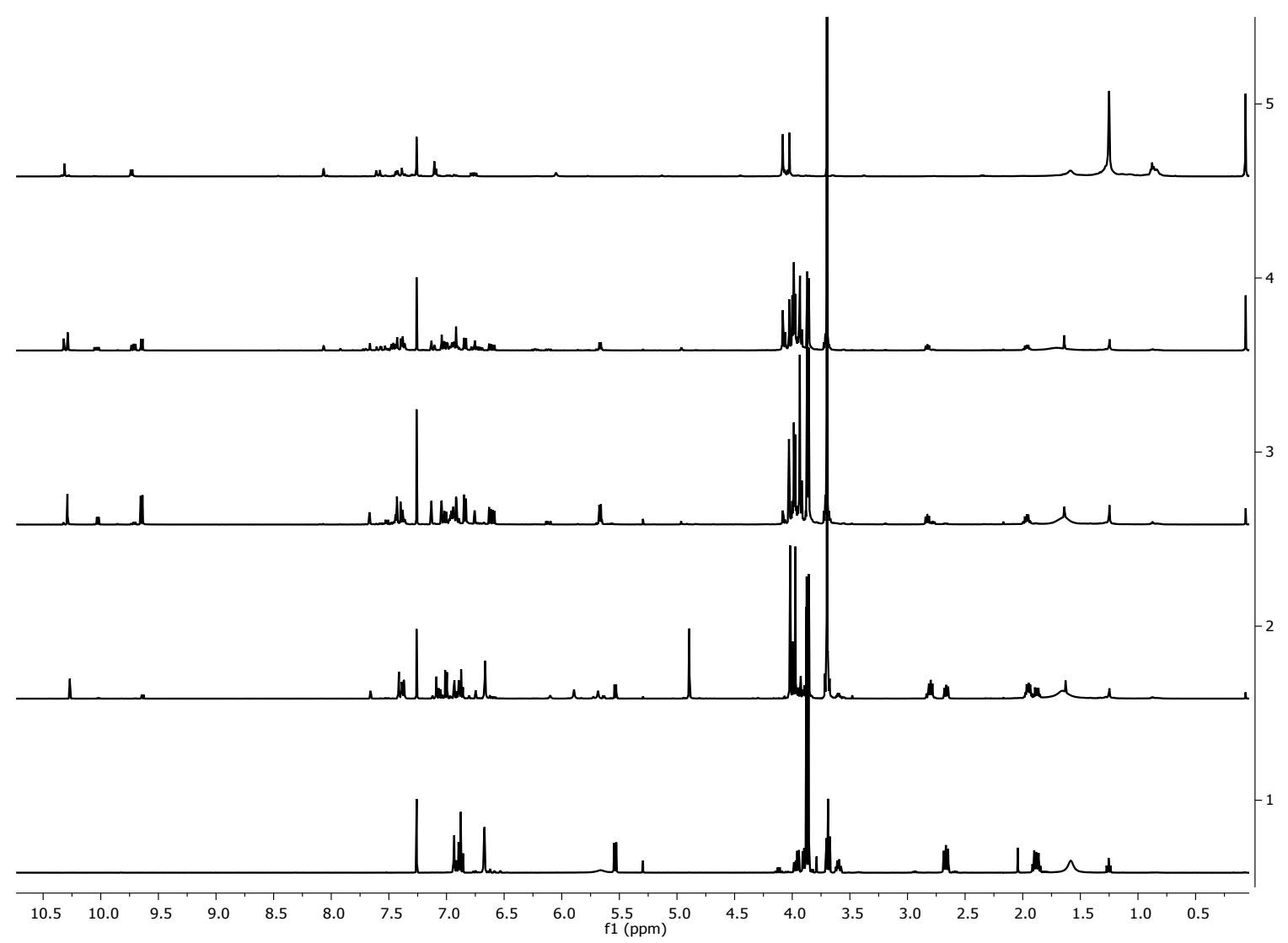

Figure S2: Crude ${ }^{1} \mathrm{H}$ NMR spectra obtained following the reaction of phenolic $\beta-5$ model compound (1) with $\mathrm{X}$ equivalents of DDQ at $60{ }^{\circ} \mathrm{C}$ in 1,4-dioxane. 1) Starting Material. 2) 1 eq. 3) 2 eq. 4) 3 eq. and 5) 10eq. 
$\underline{\text { S2.3 DDQ oxidation of model compound } 2 \text { at room temperature. }}$
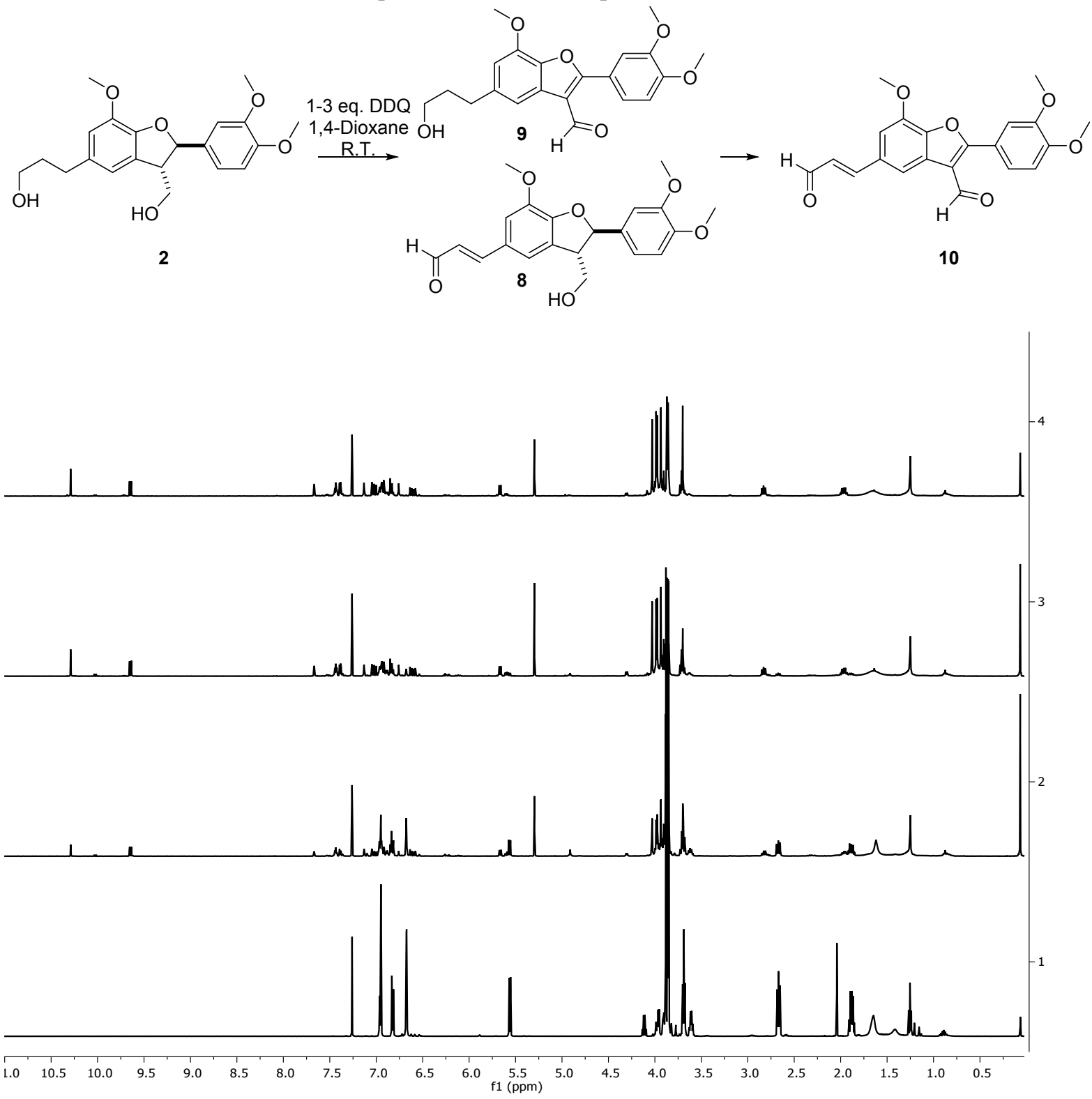

Figure S3: Crude ${ }^{1} \mathrm{H}$ NMR spectra obtained following the reaction of non-phenolic $\beta-5$ model compound (2) with $X$ equivalents of DDQ at room temperature in 1,4-dioxane. 1) Starting Material. 2) 1 eq. 3) 2 eq. and 4) 3 eq. 
$\underline{\text { S2.4 DDQ oxidation of model compound } 2 \text { at } 60^{\circ} \mathrm{C}}$
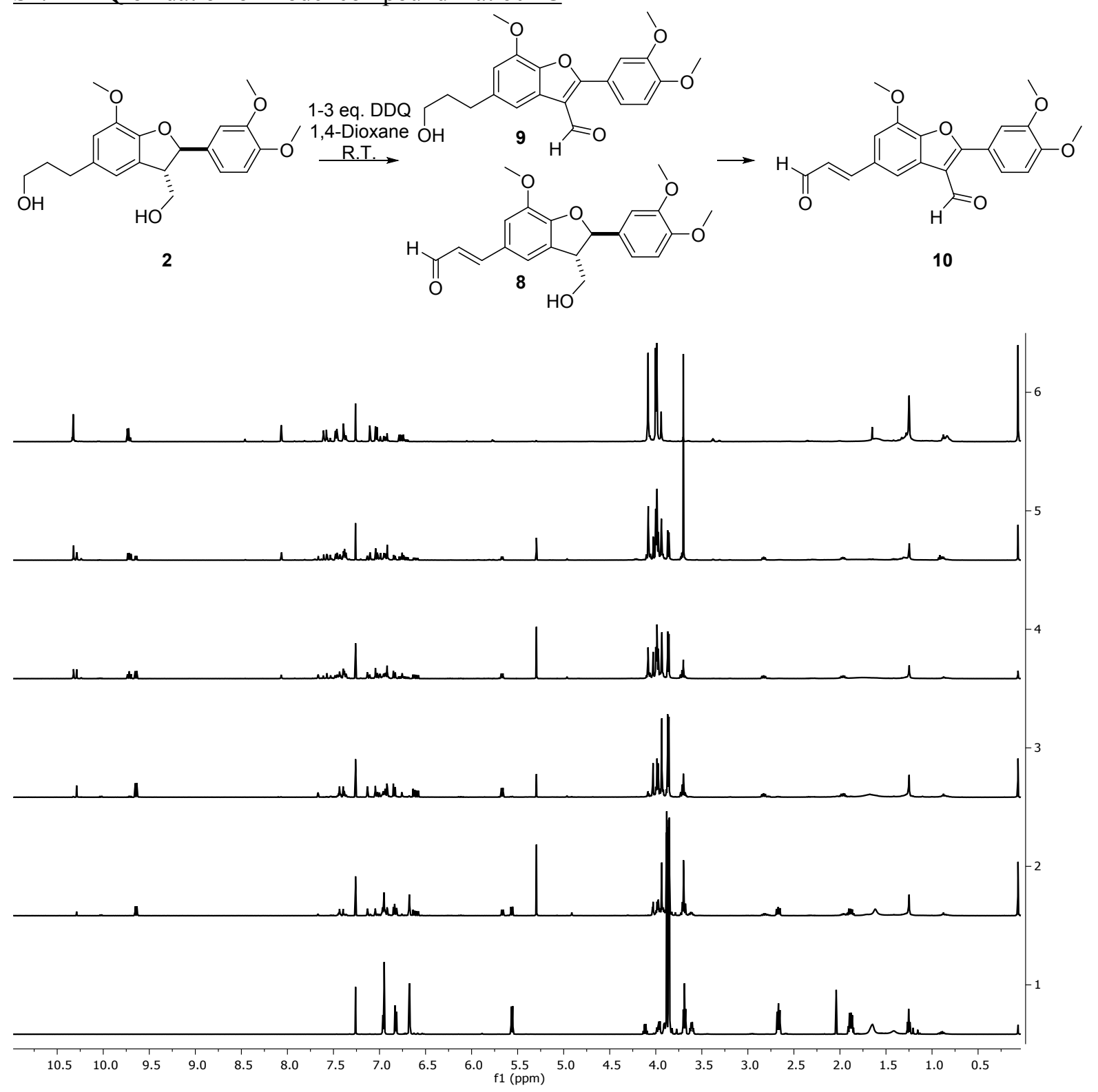

Figure S4: Crude ${ }^{1} \mathrm{H}$ NMR spectra obtained following the reaction of non-phenolic $\beta-5$ model compound (2) with $\mathrm{X}$ equivalents of DDQ at $60{ }^{\circ} \mathrm{C}$ in 1,4-dioxane. 1) Starting Material. 2) 1 eq. 3) 2 eq. and 4) 3 eq. 5) 4 eq. and 6) 10 eq. 
$\underline{\text { S2.5 DDQ oxidation of model compound } 3 \text { at room temperature. }}$<smiles>COc1cc(C2Oc3c(OC)cc(C(O)C(CO)Oc4ccccc4OC)cc3[C@@H]2CO)ccc1O</smiles>

3

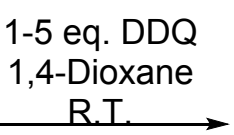<smiles>Oc1ccccc1O</smiles><smiles>COc1cc(-c2oc3c(OC)cc(C(C)=O)cc3c2C=O)ccc1O</smiles>

11

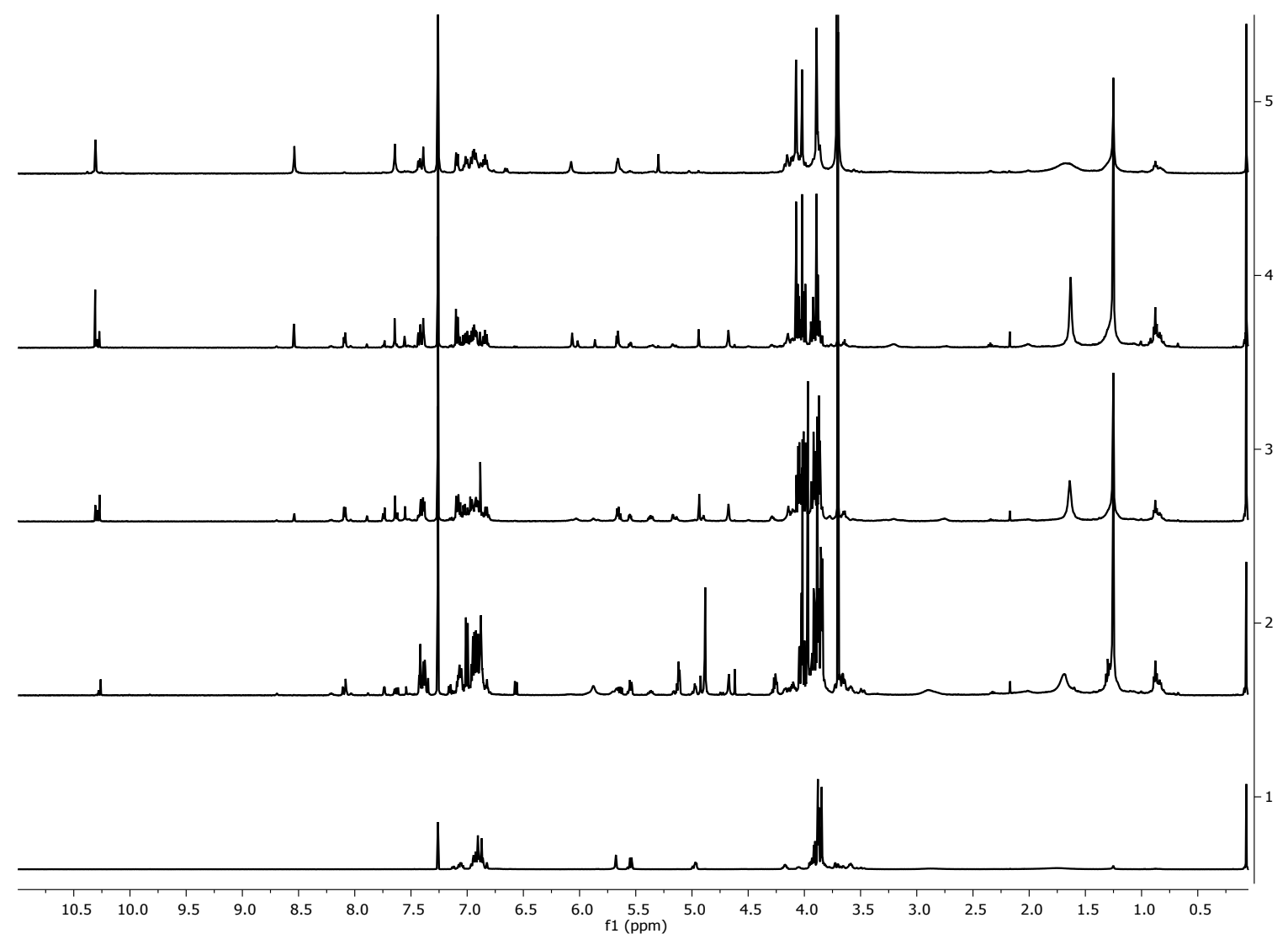

Figure S5: Crude ${ }^{1} \mathrm{H}$ NMR spectra obtained following the reaction of phenolic $\beta-\mathrm{O}-4 \beta-5$ model compound (3) with $\mathrm{X}$ equivalents of DDQ at $60^{\circ} \mathrm{C}$ in 1,4-dioxane.1) Starting Material. 2) 1 eq. 3$) 2$ eq. 4) 3 eq. and 5) 5 eq. 
$\underline{\text { S2.6 DDQ oxidation of model compound } 4 \text { at room temperature. }}$<smiles>COc1ccc([C@@H]2Oc3c(OC)cc(C(O)C(CO)Oc4ccccc4OC)cc3[C@@H]2CO)cc1OC</smiles><smiles>COc1ccc(C2Oc3c(OC)cc(C(=O)C(CO)Oc4ccccc4OC)cc3[C@@H]2CO)cc1OC</smiles>

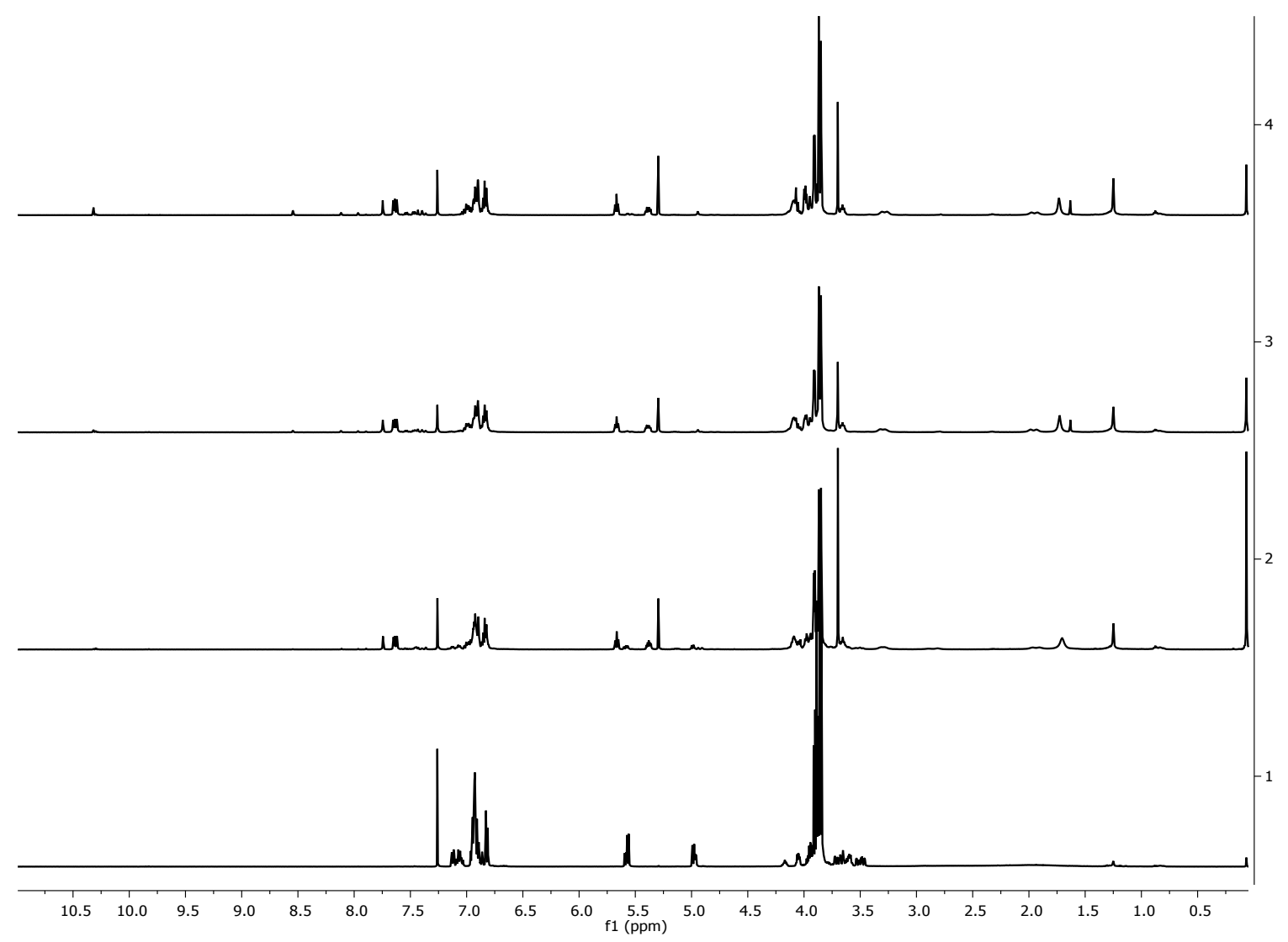

Figure S6: Crude ${ }^{1} \mathrm{H}$ NMR spectra obtained following the reaction of non-phenolic $\beta-\mathrm{O}-4 \beta-5$ model compound (4) with X equivalents DDQ at room temperature in 1,4-dioxane. 1) Starting Material. 2) 1 eq. 3) 2 eq. and 4) 3 eq. 
$\underline{\text { S2.7 DDQ oxidation of model compound } 4 \text { at } 60^{\circ} \mathrm{C}}$.<smiles>COc1ccccc1OC(CO)C(=O)c1cc(OC)c2oc(-c3ccc(OC)c(C(=O)C(CO)Oc4ccccc4OC)c3)cc2c1</smiles>

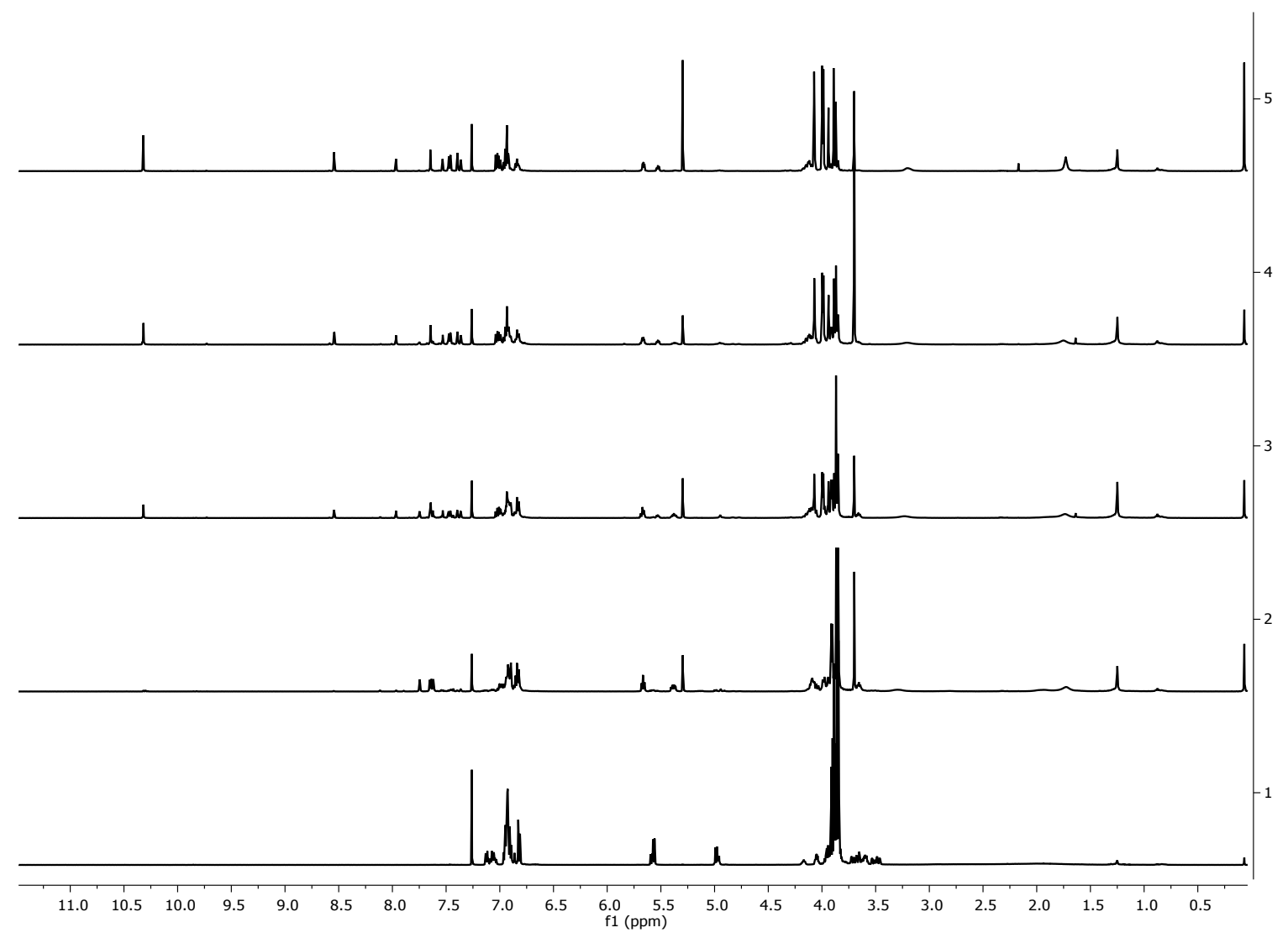

Figure S7: Crude ${ }^{1} \mathrm{H}$ NMR spectra obtained following the reaction of non-phenolic $\beta-\mathrm{O}-4 \beta-5$ model compound (4) with $\mathrm{X}$ equivalents of DDQ at $60{ }^{\circ} \mathrm{C}$ in 1,4-dioxane.1) Starting Material. 2) 1 eq. 3) 2 eq. 4) 3 eq. and 5) 4 eq. 
$\underline{\text { S3.0 Model Compound Synthesis }}$

Compounds $\mathbf{1}$ and $\mathbf{2}$ were synthesised following literature procedures. ${ }^{2}$

Compounds 3 and $\mathbf{4}$ were synthesised following literature procedures. ${ }^{3}$

Compounds 19 and 20 were synthesised from compounds 1 and 2 by acetylation with pyridine/acetic anhydride following usual procedures. ${ }^{4,5}$

$\underline{\text { S4.0 Upscaled DDQ oxidations for product isolation and charachterisation }}$

2-(4-hydroxy-3-methoxyphenyl)-5-(3-

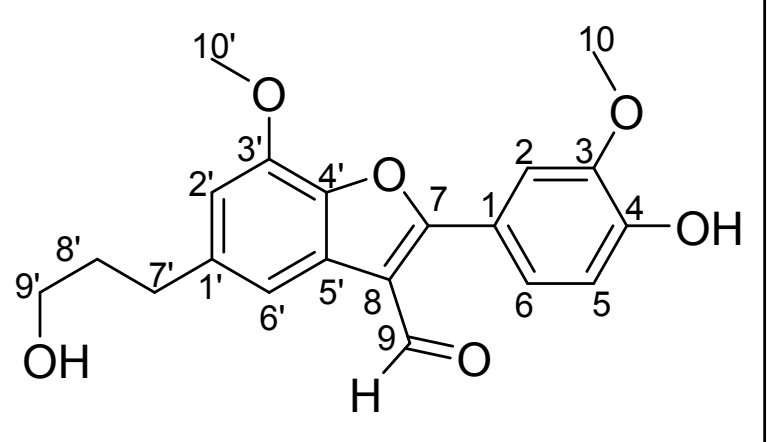
hydroxypropyl)-7-methoxybenzofuran-3carbaldehyde (5)

Synthesised following the general procedure at room temperature using compound 1 (50 $\mathrm{mg}$, $0.14 \mathrm{mmol}, 1.0$ eq.) and DDQ (95 $\mathrm{mg}, 0.42$ mmol, 3.0 eq.). Purification via column chromatography (0-60 \% EtOAc:PET Ether) allowed for the isolation of compound $\mathbf{5}$ as an off white solid (28 mg $0.078 \mathrm{mmol}, 57 \%$ ).

${ }^{1}$ H NMR (500 MHz, CDCl ) $^{2} \delta 10.28$ (s, 1H, H9), 7.67 (d, $J=1.4$ Hz, 1H, H6'), $7.43-7.36$ (m, 2H, H2/H5/H6), 7.07 (d, $J=8.2 \mathrm{~Hz}, 1 \mathrm{H}, \mathrm{H} 2 / \mathrm{H} 5 / \mathrm{H} 6), 6.75$ (d, $J=1.4 \mathrm{~Hz}, 1 \mathrm{H}, \mathrm{H} 2$ '), 6.02 (s, 1H C4$\mathrm{OH}$ ), 4.03 (s, 3H, H10'), 4.01 (s, 3H, H10'), 3.72 (t, $J=6.4 \mathrm{~Hz}, 2 \mathrm{H}, \mathrm{H} 9$ '), 2.83 (t, $J=7.7 \mathrm{~Hz}, 2 \mathrm{H}, \mathrm{H} 7$ '), $2.03-1.92(\mathrm{~m}, 2 \mathrm{H}, \mathrm{H} 8$ ').

${ }^{13}$ C NMR (126 MHz, CDCl $_{3}$ ): $\delta$ 187.0(C9), 166.2, 148.8, 147.1(C3), 144.7(C3'), 141.8, 140.1, 127.4, 123.8 (C2/C5/C6), 120.8, 116.9, 115.1 (C2/C5/C6), 113.7 (C6'), 111.2 (C2/C5/C6), 108.9 (C2'), 62.4(C9'), 56.4(C10), 56.2 (C10'), 34.9 (C8'), 32.7(C7').

HR MS: $[\mathrm{M}-\mathrm{H}]^{-} \mathrm{m} / \mathrm{z}$ calc: 355.1187 [M - H] $^{-} \mathrm{m} / \mathrm{z}$ found: 355.1185

(Data is consistent with that reported in the literature) ${ }^{6}$

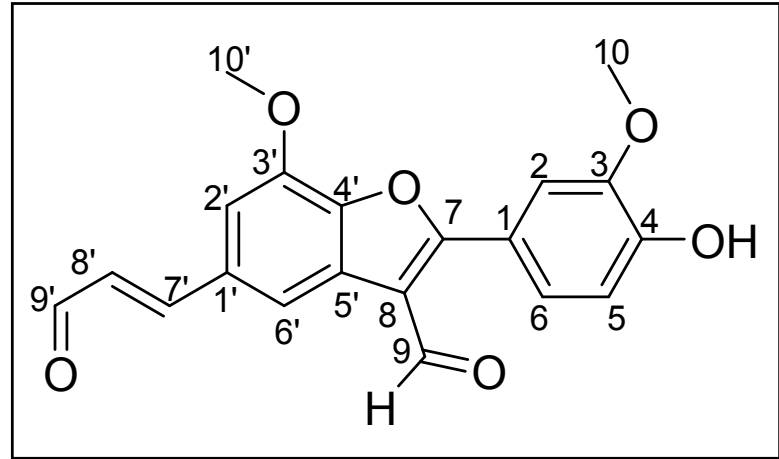

purification. 2-(4-hydroxy-3-methoxyphenyl)-7-methoxy-5(3-oxoprop-1-en-1-yl)benzofuran-3carbaldehyde (7)

Synthesised following the general procedure at $60{ }^{\circ} \mathrm{C}$ using compound $1(100 \mathrm{mg}, 0.14 \mathrm{mmol}$, 1.00 eq.) and DDQ (630 mg, $2.78 \mathrm{mmol}, 10.0$ eq.). Purification via column chromatography (0$80 \%$ EtOAc:PET Ether) allowed for the isolation of compound 7 as a white solid (16.6 mg 0.047 mmol, 17\%). Crude NMR analysis (Figure S2) suggested quite a clean reaction and so the low yield is attributed to losses during work-up and

${ }^{1} \mathbf{H}$ NMR (400 MHz, CDCl 3 ): $\delta 10.32(\mathrm{~s}, 1 \mathrm{H}),, 9.74(\mathrm{~d}, J=7.6 \mathrm{~Hz}, 1 \mathrm{H}), 8.07(\mathrm{~d}, J=1.5 \mathrm{~Hz}, 1 \mathrm{H}), 7.60$ (d, $J=15.9 \mathrm{~Hz}, 1 \mathrm{H}), 7.44(\mathrm{dd}, J=8.2,2.0 \mathrm{~Hz}, 1 \mathrm{H}), 7.39(\mathrm{~d}, J=2.0 \mathrm{~Hz}, 1 \mathrm{H}), 7.13-7.08(\mathrm{~m}, 2 \mathrm{H}), 6.77$ (dd, $J=15.9,7.6 \mathrm{~Hz}, 1 \mathrm{H}), 6.02(\mathrm{~s}, 1 \mathrm{H}), 4.08(\mathrm{~s}, 3 \mathrm{H}), 4.03(\mathrm{~s}, 3 \mathrm{H})$.

${ }^{13}$ C NMR (126 MHz, $\mathbf{C D C l}_{3}$ ) $\delta 193.8,186.6,166.7,153.2,149.2,147.2,145.5,144.8,132.3,128.6$, $128.1,124.0,120.2,116.9,116.7,115.3,111.1,106.7,56.5,56.3$.

HR MS: $[\mathrm{M}-\mathrm{H}]^{-} \mathrm{m} / \mathrm{z}$ calc: $351.0874[\mathrm{M}-\mathrm{H}]^{-} \mathrm{m} / \mathrm{z}$ found: 351.0873

(Data is consistent with that reported in the literature) ${ }^{7}$ 


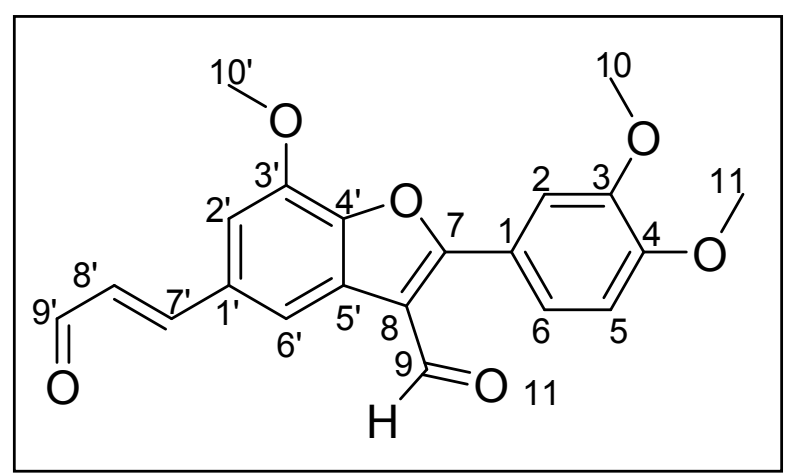

2-(3,4-dimethoxyphenyl)-7-methoxy-5-(3oxoprop-1-en-1-yl)benzofuran-3-carbaldehyde (10)

Synthesised following the general procedure at $60{ }^{\circ} \mathrm{C}$ using compound $2(100 \mathrm{mg}, 0.27 \mathrm{mmol}$, 1.0 eq.) and DDQ (606 mg, $2.67 \mathrm{mmol}, 10.0$ eq.). Purification via column chromatography $(0-60 \%$ EtOAc:PET Ether) allowed for the isolation of compound $\mathbf{1 0}$ as a white solid (39 $\mathrm{mg}, 0.11$ mmol, 44\%).

${ }^{1} \mathbf{H}$ NMR (500 MHz, CDCl $)$ ): $\delta 10.33$ (s, 1H, H9), 9.74 (d, $J=7.7 \mathrm{~Hz}, 1 \mathrm{H}, \mathrm{H} 9$ '), 8.07 (d, $J=1.4 \mathrm{~Hz}$, 1H, H2'/H6'), 7.60 (d, $J=15.9 \mathrm{~Hz}, 1 \mathrm{H}, \mathrm{H} 7$ '), 7.47 (dd, $J=8.4,2.1 \mathrm{~Hz}, 1 \mathrm{H}, \mathrm{H6}$ ), 7.39 (d, $J=2.1 \mathrm{~Hz}$, 1H, H2/H5), 7.11 (d, $J=1.4 \mathrm{~Hz}, 1 \mathrm{H}, \mathrm{H} 2$ '/H6'), 7.04 (d, $J=8.4 \mathrm{~Hz}, 1 \mathrm{H}, \mathrm{H} 2 / \mathrm{H} 5), 6.77$ (dd, $J=15.9,7.7$ $\mathrm{Hz}, 1 \mathrm{H}, \mathrm{H} 8$ '), 4.09 (s, 3H, $\left.-\mathrm{OC}_{3}\right), 4.00$ (s, $\left.3 \mathrm{H},-\mathrm{OC}_{3}\right), 3.99$ (s, 3H, $\left.-\mathrm{OC}_{3} \underline{H}_{3}\right)$.

${ }^{13}$ C NMR (126 MHz, CDCl $)_{3}$ : $\delta 193.8$ (C9), 186.6 (C9'), 166.6 (C7), 153.2 (H7'), 152.2 (C3/C4), 149.7 (C3/C4), 145.5 (C3'), 144.8, 132.3 (C1'), 128.6 (C8'), 128.1, 123.3 (C6), 120.7, 116.9 (C2'/C6'), 116.8, $111.5(\mathrm{C} 2 / \mathrm{C} 5), 111.4(\mathrm{C} 2 / \mathrm{C} 5), 106.7\left(\mathrm{C}^{\prime} / \mathrm{C} 6\right.$ ') $56.4\left(-\mathrm{OCH}_{3}\right), 56.33\left(-\mathrm{OCH}_{3}\right), 56.29\left(-\mathrm{OCH}_{3}\right)$.

HR MS: $[\mathrm{M}+\mathrm{H}]^{+} \mathrm{m} / \mathrm{z}$ calc: $367.1176[\mathrm{M}+\mathrm{H}]^{+} \mathrm{m} / \mathrm{z}$ found: 367.1179

IR: (thin film) 1684, 1670, 1653, 1559, 1541, 1506, 1474, 1456, 1026.

(Data is consistent with that reported in the literature) ${ }^{8}$

5-(3-hydroxy-2-(2-methoxyphenoxy)propanoyl)-2-(4-hydroxy-3-methoxyphenyl)-7-

methoxybenzofuran-3-carbaldehyde (11)

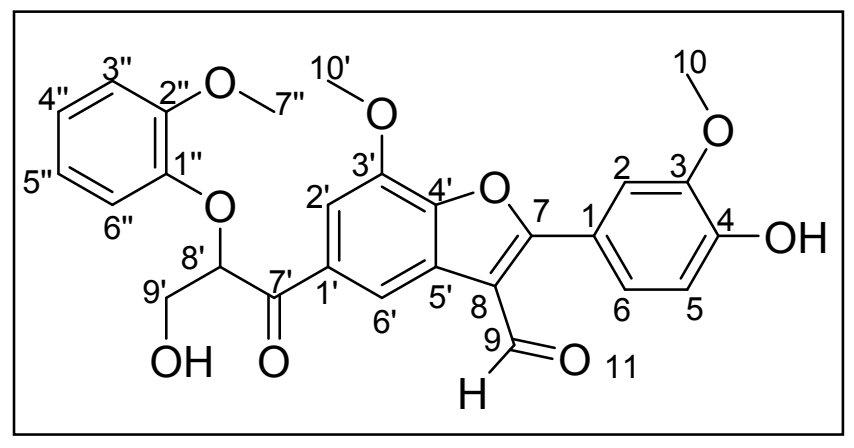

Compound 3 (100 mg $0.201 \mathrm{mmol} 1.00 \mathrm{eq}$ ) was dissolved in dioxane $(2.95 \mathrm{ml})$ and DDQ (227 mg, $1.00 \mathrm{mmol} 5.00 \mathrm{eq}$ ) was added and the reaction allowed to proceed at room temperature for 24 hours. The reaction was then filtered through a cotton plug. Due to the products solubility in $\mathrm{H}_{2} \mathrm{O}$ aqueous extraction of residual DDQ/DDQH 2 was not possible. The organic layer was concentrated in vacuo prior to being dissolved in DCM and transferred directly to a silica chromatography column. The column was eluted with 1-5\% MeOH/DCM which allowed isolation of compound $\mathbf{1 1}$ as a yellow solid (29.1 $\mathrm{mg} 0.598 \mathrm{mmol}, 30 \%)$.

${ }^{1}$ H NMR (500 MHz, CDCl $): \delta 10.31(\mathrm{~s}, 1 \mathrm{H}, \mathrm{H} 9), 8.53(\mathrm{~d}, J=1.5 \mathrm{~Hz}, 1 \mathrm{H}, \mathrm{H} 6$ ') $7.64(\mathrm{~d}, J=1.5 \mathrm{~Hz}$, $1 \mathrm{H}, \mathrm{H} 2$ ') 7.42 (dd, $J=8.2,1.9 \mathrm{~Hz}, 1 \mathrm{H}, \mathrm{H} 6), 7.38$ (d, $J=2.0 \mathrm{~Hz}, 1 \mathrm{H}, \mathrm{H} 2 / \mathrm{H} 5), 7.09$ (d, $J=8.2 \mathrm{~Hz}, 1 \mathrm{H}$, H2/H5), 7.02 (ddd, $J=8.1,7.4,1.6 \mathrm{~Hz}, 1 \mathrm{H}, \mathrm{Ar}-\underline{\mathrm{H}}$ ), 6.95 (ddd, $J=15.1,8.1,1.6 \mathrm{~Hz}, 2 \mathrm{H}, \mathrm{Ar}-\underline{\mathrm{H}}$ ), 6.84 (ddd, $J=8.0,7.4,1.6 \mathrm{~Hz}, 1 \mathrm{H}, \mathrm{Ar}-\underline{\mathrm{H}}), 6.08$ (s, $1 \mathrm{H}, \mathrm{C} 4-\mathrm{OH}), 5.67$ (dd, $J=5.8,3.5 \mathrm{~Hz}, 1 \mathrm{H}, \mathrm{H} 8$ '), 4.19 4.09 (m, 2H, H9'), 4.07 (s, 3H, - $\left.\mathrm{OCH}_{3}\right), 4.02\left(\mathrm{~s}, 3 \mathrm{H},-\mathrm{OC}_{3}\right), 3.89$ (s, $\left.3 \mathrm{H},-\mathrm{OCH}_{3}\right)$.

${ }^{13}$ C NMR (101 MHz, CDCl 3 ): $\delta 196.1$ (C9), 186.4 (C7'), 166.9 (C4), 150.8, 149.3, 147.2, 146.8, 146.1, 145.6, 133.3, 127.4, 123.97 (C6), 123.92, 121.3, 120.1, 118.9, 116.8 (C8), 116.5 (C6'), 115.3 (C2/C5),

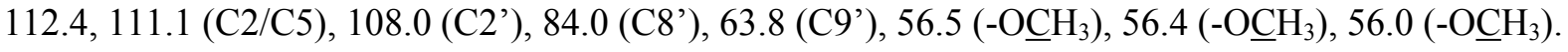
HR MS: [M - H] ${ }^{-} \mathrm{m} / \mathrm{z}$ calc: $491.1348[\mathrm{M}-\mathrm{H}]^{-} \mathrm{m} / \mathrm{z}$ found: 491.1348

IR: (thin film) 1684, 1653, 1593, 1506, 1254, 1161, 1126, 1020.

MP: $88-90{ }^{\circ} \mathrm{C}$ 
1-(2-(3,4-Dimethoxyphenyl)-3-(hydroxymethyl)-7-methoxy-2,3-dihydrobenzofuran-5-yl)-3-hydroxy2-(2-methoxyphenoxy)propan-1-one (13)

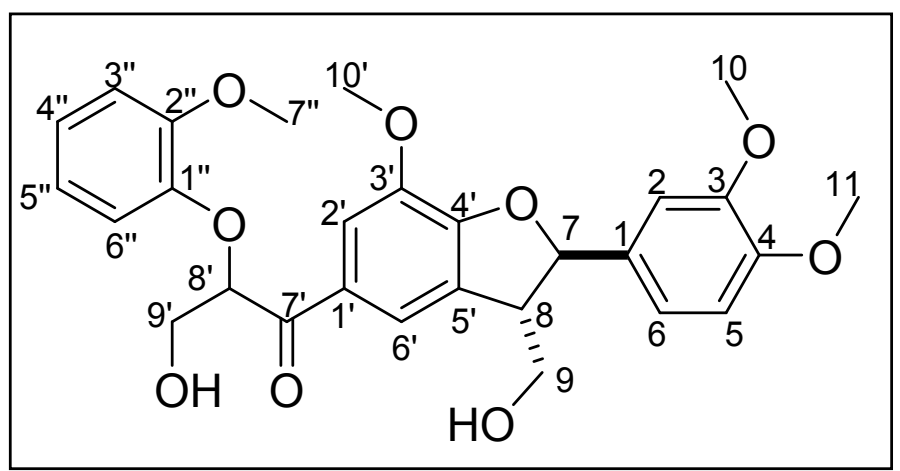

Synthesised following the general procedure at room temperature using compound 4 (200 mg, $0.390 \mathrm{mmol} 1.00$ eq.) and DDQ (106 mg $0.780 \mathrm{mmol}, 2.00$ eq.). Purification via column chromatography (10-70\% Acetone:PET Ether) allowed for the isolation of compound $\mathbf{1 3}$ as a pale yellow solid (137 mg $0.269 \mathrm{mmol}, 69 \%$, mixture of racemic diastereomers).

${ }^{1} \mathbf{H}$ NMR (500 MHz, CDCl 3$): \delta 7.80-7.57(\mathrm{~m}, 2 \mathrm{H}, \mathrm{Ar}-\underline{\mathrm{H}}), 7.01-6.80(\mathrm{~m}, 7 \mathrm{H}, \mathrm{Ar}-\underline{\mathrm{H}}), 5.71-5.62(\mathrm{~m}$, 1H, H7), $5.46-5.34$ (m, 1H, H8'), 4.08 (q, $J=14.7,11.1 \mathrm{~Hz}, 2 \mathrm{H}, \mathrm{H} 9$ '), $4.01-3.93$ (m, 2H, H9), 3.91 $\left(\mathrm{s}, 1.5 \mathrm{H},-\mathrm{OCH}_{3}\right), 3.90\left(\mathrm{~s}, 1.5 \mathrm{H},-\mathrm{OC}_{3}\right), 3.88-3.82\left(\mathrm{~m}, 9 \mathrm{H},-\mathrm{OC}_{3}\right), 3.69-3.61(\mathrm{~m}, 1 \mathrm{H}, \mathrm{H} 8)$.

${ }^{13}$ C NMR (126 MHz, CDCl 3 ): $\delta 195.2$ (C7'), 194.9 (C7'), 153.7, 150.3, 150.1, 149.42, 149.36, 146.93, $146.85,144.69,132.7,132.6,129.3,129.2,128.53,128.47,123.6,123.4,121.4,119.14,119.09,119.0$, 118.9, 117.9, 117.3, 113.18, 113.16, 112.5, 111.2, 110.1, 109.4, 107.6, 89.4 (C7), 84.3 (C8'), 84.2 (C8'),

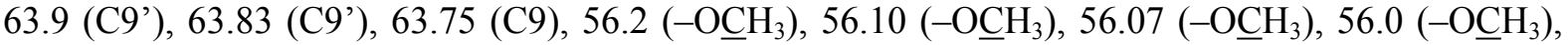
$53.0(\mathrm{C} 8)$.

${ }^{1}$ H NMR (700 MHz, DMSO-d d): $^{\delta} 7.74$ (s, 1H, H2'/H6'), 7.60 (s, 1H, H2'/H6'), 6.99 - 6.94 (m, 3H, Ar- -6$), 6.89(\mathrm{~m}, 2 \mathrm{H}, \mathrm{Ar}-\underline{\mathrm{H}}), 6.80-6.74(\mathrm{~m}, 2 \mathrm{H}, \mathrm{Ar}-\underline{\mathrm{H}}), 5.67-5.59$ (m, 2H, H7 + H8'), 5.20 (m, 1H,

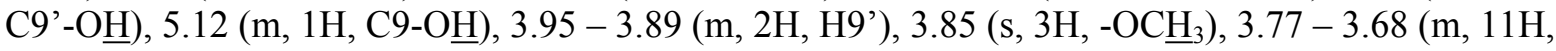
$\left.3 \mathrm{x}-\mathrm{OC} \underline{\mathrm{H}}_{3}+\mathrm{H} 9\right), 3.59-3.54(\mathrm{~m}, 1 \mathrm{H}, \mathrm{H} 8)$.

${ }^{13}$ C NMR (176 MHz, DMSO-d $\left.\mathbf{d}_{\mathbf{6}}\right): \delta 194.8$ (C7'), 194.7 (C7'), 152.5, 149.2, 149.1, 148.8, 146.9, 146.9, 143.6, 133.0, 129.7, 129.7, 128.9, 121.6, 121.5, 120.5, 118.9, 118.4, 114.5, 114.3, 112.8, 112.7, 112.6, 111.7, 109.9, 88.4 (C7), 81.5 (C8'), 81.4 (C8'), 62.6 (C9/C9'), 62.5 (C9/C9'), 55.8 (- $\left.\mathrm{OC}_{3}\right)$, $55.6\left(-\mathrm{OCH}_{3}\right), 55.5\left(-\mathrm{OCH}_{3}\right), 52.4\left(-\mathrm{OCH}_{3}\right)$.

HR MS: $[\mathrm{M}+\mathrm{H}]^{+} \mathrm{m} / \mathrm{z}$ calc: $511.1963[\mathrm{M}+\mathrm{H}]^{+} \mathrm{m} / \mathrm{z}$ found: 511.1958

IR: (thin film) $1684,1591,1506,1456,1254,1157,1126,1020$.

MP: $74-76{ }^{\circ} \mathrm{C}$

1-(2-(3,4-Dimethoxyphenyl)-3-(hydroxymethyl)-7-methoxy-2,3-dihydrobenzofuran-5-yl)-3-hydroxy2-(2-methoxyphenoxy)propan-1-one (14) and 1-(2-(3,4-dimethoxyphenyl)-7-methoxybenzofuran-5-

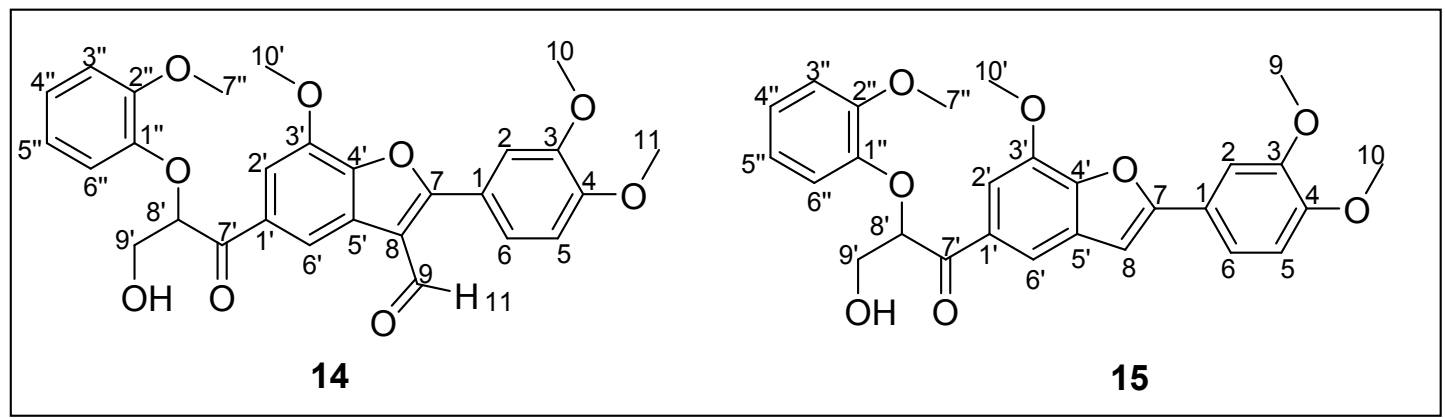

yl)-3-hydroxy-2-(2-methoxyphenoxy)propan-1-one (15)

Synthesised following the general procedure at $60{ }^{\circ} \mathrm{C}$ using compound $4(200 \mathrm{mg}, 0.390 \mathrm{mmol}, 1.00$ eq.) and DDQ (443 mg, $1.95 \mathrm{mmol}, 5.0$ eq.). Purification via preparative TLC allowed for the isolation 
of compound 14 as an off white solid (76 $\mathrm{mg} 0.15 \mathrm{mmol}, 38 \%$ ) and compound 15 as an off white solid (43 mg $0.090 \mathrm{mmol}, 23 \%$ )

\section{Compound 14}

${ }^{1} \mathbf{H}$ NMR (500 MHz, CDCl $\mathbf{~}_{3}$ ): $\delta 10.32$ (s, 1H, H9), 8.55 (d, $J=1.5 \mathrm{~Hz}, 1 \mathrm{H}, \mathrm{H} 2$ '/H6'), 7.65 (d, $J=1.6$ Hz, 1H, H2'/H6'), 7.47 (dd, $J=8.3,2.1 \mathrm{~Hz}, 1 \mathrm{H}, \mathrm{Ar}-\underline{\mathrm{H}}$ ), 7.40 (d, $J=2.1 \mathrm{~Hz}, 1 \mathrm{H}, \mathrm{Ar}-\underline{\mathrm{H}}), 7.05-7.02$ (m, $1 \mathrm{H}, \operatorname{Ar}-\underline{\mathrm{H}}), 7.02-6.99(\mathrm{~m}, 1 \mathrm{H}, \mathrm{Ar}-\underline{\mathrm{H}}), 6.97-6.95(\mathrm{~m}, 1 \mathrm{H}, \mathrm{Ar}-\underline{\mathrm{H}}), 6.94-6.92$ (m, 1H, Ar- $\underline{\mathrm{H}}), 6.86-$ $6.82(\mathrm{~m}, 1 \mathrm{H}, \mathrm{Ar}-\underline{\mathrm{H}}), 5.66$ (dd, $\left.J=6.0,3.5 \mathrm{~Hz}, 1 \mathrm{H}, \mathrm{H} 8^{\prime}\right), 4.18-4.09$ (m, 2H, H9;), 4.08 (s, 3H, C10'), $4.00\left(\mathrm{~s}, 3 \mathrm{H},-\mathrm{OC} \underline{\mathrm{H}}_{3}\right), 3.99\left(\mathrm{~s}, 3 \mathrm{H},-\mathrm{OC}_{3}\right), 3.90\left(\mathrm{~s}, 3 \mathrm{H},-\mathrm{OC} \underline{H}_{3}\right)$.

${ }^{13}$ C NMR (126 MHz, CDCl 3 ): $\delta 196.1$ (C7'), 186.4 (C9), 166.8, 152.3, 150.8, 149.7, 146.9, 146.1, 145.6 (C3'), 133.3, 129.2, 128.4, 127.4, 125.4, 123.9, 123.3, 121.3, 120.6, 118.9, 118.6, 117.0, 116.5, $112.4,111.5,111.4,108.0,106.3,84.1\left(\mathrm{C} 8\right.$ '), 63.8 (C9'), $56.4\left(\mathrm{C} 10^{\prime}\right), 56.3\left(-\mathrm{OC}_{3}\right), 56.1\left(-\mathrm{OCH}_{3}\right)$, $56.0\left(-\mathrm{OCH}_{3}\right)$.

${ }^{1}$ H NMR (700 MHz, DMSO-d $\left.)_{6}\right): \delta 10.28(\mathrm{~s}, 1 \mathrm{H}), 8.48(\mathrm{~d}, J=1.4 \mathrm{~Hz}, 1 \mathrm{H}), 7.70(\mathrm{~d}, J=1.4 \mathrm{~Hz}, 1 \mathrm{H})$, $7.62(\mathrm{dd}, J=8.4,2.1 \mathrm{~Hz}, 1 \mathrm{H}), 7.55(\mathrm{~d}, J=2.1 \mathrm{~Hz}, 1 \mathrm{H}), 7.21(\mathrm{~d}, J=8.5 \mathrm{~Hz}, 1 \mathrm{H}), 6.98(\mathrm{dd}, J=8.1,1.4$ $\mathrm{Hz}, 1 \mathrm{H}), 6.90$ (td, $J=7.7,1.6 \mathrm{~Hz}, 1 \mathrm{H}), 6.84$ (dd, $J=8.1,1.6 \mathrm{~Hz}, 1 \mathrm{H}), 6.79$ (td, $J=7.7,1.5 \mathrm{~Hz}, 1 \mathrm{H})$, $5.72(\mathrm{t}, J=5.0 \mathrm{~Hz}, 1 \mathrm{H}), 5.31(\mathrm{t}, J=5.7 \mathrm{~Hz}, 1 \mathrm{H}), 4.05(\mathrm{~s}, 3 \mathrm{H}), 3.97(\mathrm{t}, J=5.3 \mathrm{~Hz}, 2 \mathrm{H}), 3.90(\mathrm{~s}, 3 \mathrm{H})$, $3.89(\mathrm{~s}, 3 \mathrm{H}), 3.74(\mathrm{~s}, 3 \mathrm{H})$.

${ }^{13}$ C NMR (176 MHz, DMSO- $\boldsymbol{d}_{6}$ ): $\delta$ 196.6, 186.72, 165.69, 151.9, 149.4, 149.1, 146.8, 144.9, 144.7, $133.4,127.0,122.9,122.0,120.6,119.7,116.0,115.3,115.2,112.7,112.1,111.7,108.1,81.9,62.5$, $56.1,55.9,55.8,55.5$.

HR MS: $\left[\mathrm{M}+\mathrm{H}^{+}\right] \mathrm{m} / \mathrm{z}$ calc: $507.1650\left[\mathrm{M}+\mathrm{H}^{+}\right] \mathrm{m} / \mathrm{z}$ found: 507.1640

IR: (thin film) 1659, 1595, 1503, 1260, 1126, 1017.

MP: $82-84{ }^{\circ} \mathrm{C}$

\section{Compound 15}

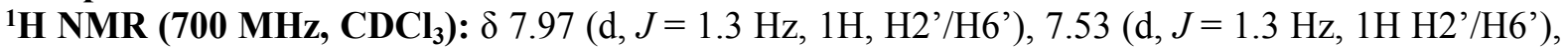
7.47 (dd, $J=8.4,1.9 \mathrm{~Hz}, 1 \mathrm{H}), 7.36(\mathrm{~d}, J=2.0 \mathrm{~Hz}, 1 \mathrm{H}), 7.01$ (td, $J=7.8,1.5 \mathrm{~Hz}, 1 \mathrm{H}), 6.97-6.89$ (m, $4 \mathrm{H}), 6.83$ (td, $J=7.7,1.4 \mathrm{~Hz}, 1 \mathrm{H}), 5.52$ (dd, $J=6.6,4.0 \mathrm{~Hz}, 1 \mathrm{H}, \mathrm{H} 8$ '), $4.15-4.09$ (m, 2H, H9'), 4.07 $\left(\mathrm{s}, 3 \mathrm{H},-\mathrm{OCH}_{3}\right), 3.99\left(\mathrm{~s}, 3 \mathrm{H},-\mathrm{OC}_{3}\right), 3.94\left(\mathrm{~s}, 3 \mathrm{H},-\mathrm{OC}_{3}\right), 3.87\left(\mathrm{~s}, 3 \mathrm{H},-\mathrm{OC} \underline{\mathrm{H}}_{3}\right)$.

${ }^{13}$ C NMR (176 MHz, CDCl ${ }_{3}$ ): $\delta 196.0$ (C7'), 158.0, 150.7, 150.2, 149.4, 147.2, 147.1, 145.6, 131.4, 131.0, 123.9, 122.7, 121.3, 118.9, 118.5, 115.8, 112.4, 111.4, 108.3, 106.3, 100.8, 84.9 (C8'), 64.0 (C9'), $56.30\left(-\mathrm{OCH}_{3}\right), 56.25\left(-\mathrm{OCH}_{3}\right), 56.1\left(-\mathrm{OCH}_{3}\right), 56.0\left(-\mathrm{OCH}_{3}\right)$.

${ }^{1} \mathbf{H}$ NMR (700 MHz, DMSO-d $\left.\mathbf{d}_{\mathbf{6}}\right): \delta 8.11$ (d, $J=1.4 \mathrm{~Hz}, 1 \mathrm{H}, \mathrm{H} 2$ '/H6'), $7.54-7.42$ (m, 4H. Ar- $\left.\underline{\mathrm{H}}\right), 7.10$ $(\mathrm{d}, J=8.4 \mathrm{~Hz}, 1 \mathrm{H}, \mathrm{Ar}-\underline{\mathrm{H}}), 6.97(\mathrm{dd}, J=8.1,1.4 \mathrm{~Hz}, 1 \mathrm{H}, \operatorname{Ar}-\underline{\mathrm{H}}), 6.88(\mathrm{td}, J=7.7,1.7 \mathrm{~Hz}, 1 \mathrm{H}, \operatorname{Ar}-\underline{\mathrm{H}})$, $6.83-6.75(\mathrm{~m}, 2 \mathrm{H}, \mathrm{Ar}-\underline{\mathrm{H}}), 5.78\left(\mathrm{t}, J=4.9 \mathrm{~Hz}, 1 \mathrm{H}, \mathrm{H} 8^{\prime}\right), 5.24(\mathrm{t}, J=5.8 \mathrm{~Hz}, 1 \mathrm{H} \mathrm{C9}-\mathrm{O} \underline{\mathrm{H}}), 4.02(\mathrm{~s}, 3 \mathrm{H}$, $\left.-\mathrm{OC}_{3}\right), 3.97-3.93\left(\mathrm{~m}, 2 \mathrm{H}, \mathrm{H} 9\right.$ '), $3.88\left(\mathrm{~s}, 3 \mathrm{H},-\mathrm{OC}_{3}\right), 3.82\left(\mathrm{~s}, 3 \mathrm{H},-\mathrm{OC}_{3}\right), 3.75\left(\mathrm{~s}, 3 \mathrm{H},-\mathrm{OC}_{3}\right)$.

${ }^{13}$ C NMR (176 MHz, DMSO-d $\boldsymbol{d}_{6}$ ): $\delta 196.2$ (C7'), 157.1, 149.9, 149.22, 149.1, 146.9, 145.9, 144.7, 131.8, 130.6, 121.9, 121.7, 120.6, 117.9, 115.4, 114.7, 112.7, 112.1, 108.3, 106.1, 101.5, 81.6 (C8'), $62.6(\mathrm{C} 9 '), 55.9\left(-\mathrm{OCH}_{3}\right), 55.7\left(-\mathrm{O}^{\prime} H_{3}\right), 55.6\left(-\mathrm{O}^{\prime} H_{3}\right), 55.5\left(-\mathrm{O}^{-} \mathrm{H}_{3}\right)$.

HR MS: $\left[\mathrm{M}+\mathrm{H}^{+}\right] \mathrm{m} / \mathrm{z}$ calc: $479.1700\left[\mathrm{M}+\mathrm{H}^{+}\right] \mathrm{m} / \mathrm{z}$ found: 479.1692

IR: (thin film) 1684, 1506, 1456, 1252, 1217, 1165, 1018.

MP: $78-81{ }^{\circ} \mathrm{C}$

$\underline{\text { S5.0 Oxidation of model softwood lignin polymer. }}$

To a lignin model polymer $\mathbf{S} 1$ containing $\beta-0-4, \beta-\beta$ and $\beta-5$ units (unit ratio 74:9:17, $26 \mathrm{mg})^{9}$ in 1,4dioxane/methanol (9:1, $0.50 \mathrm{~mL})$ was added DDQ $(26 \mathrm{mg})$. The reaction mixture was stirred for 16 hours, filtered through a plug of cotton wool and precipitated in $\mathrm{Et}_{2} \mathrm{O}(10 \mathrm{~mL})$. The polymer was then collected by filtration and dried in vacuo to give the oxidised model polymer $\mathbf{S 2}$ as a brown powder ( $26 \mathrm{mg}$ ). See Figure S10 for HSQC NMR analysis. 


\section{$\underline{\text { S6.0 Lignin Experimental }}$}

\section{S6.1 Lignin Extraction Procedure}

Douglas fir sawdust (300 g) was added to 1,4-dioxane: $0.5 \mathrm{M} \mathrm{HCl}_{\mathrm{aq}}(8: 2, \sim 10 \mathrm{~mL} / \mathrm{g}$, overall $0.1 \mathrm{M} \mathrm{HCl})$. This was heated at gentle reflux for $1.5 \mathrm{hr}$ and then filtered after cooling. The filter cake was washed with a mixture of 1,4-dioxane:water (8:2). The filtrate was concentrated in vacuo giving a brown/red gum. The gum-like material was then dissolved in a minimum amount of acetone: $\mathrm{H}_{2} \mathrm{O}$ (ca. 9:1) and added dropwise to vigorously stirred $\mathrm{H}_{2} \mathrm{O}$ (at least 10x volume). The precipitate was collected (filtration), washed well with water, and dried in a desiccator over $\mathrm{CaCl}_{2}$. The dried precipitate was then dissolved in acetone: $\mathrm{MeOH}$ (9:1, 1 volume) and added dropwise to diethyl ether (10x volume). Precipitated dioxasolv lignin was further reprecipitated from acetone:MeOH (9:1, 1 volume) into ethyl acetate (10x volume) and dried in a vacuum oven overnight prior to analysis.

\section{S6.2 Lignin Oxidation Procedure}

DDQ (1-2 weight equivalent) was added to a solution of Douglas fir dioxosolv lignin $(100 \mathrm{mg})$ in dioxane $(6 \mathrm{~mL})$. The mixture was then stirred at room temperature $/ 60{ }^{\circ} \mathrm{C}$ for 24 hours. The reaction mixture was centrifuged to remove $\mathrm{DDQH}_{2}$ after which the supernatant was removed and then added to diethyl ether $(100 \mathrm{~mL})$ to induce precipitation of lignin. The mixture was then centrifuged and the precipitated lignin was collected before being dried in the desiccator under vacuum.

\section{$\underline{\text { S6.3 Lignin Acetylation Procedure }}$}

To Douglas fir dioxasolv lignin $(100 \mathrm{mg})$ was added pyridine $(1.5 \mathrm{~mL})$ and acetic anhydride $(1.5 \mathrm{~mL})$. The mixture was then stirred at room temperature for 16 hours. The reaction mixture was then quenched with ethanol and concentrated in vacuo. The product was dissolved in ethanol and concentrated in vacuo a further 4 times. The product was then dissolved in chloroform and washed sequentially with $1 \mathrm{M} \mathrm{HCl}$, a saturated solution of $\mathrm{NaHCO}_{3}$ and an EDTA solution. The organic layer was then dried over $\mathrm{MgSO}_{4}$, filtered and dried in vacuo prior to analysis. 


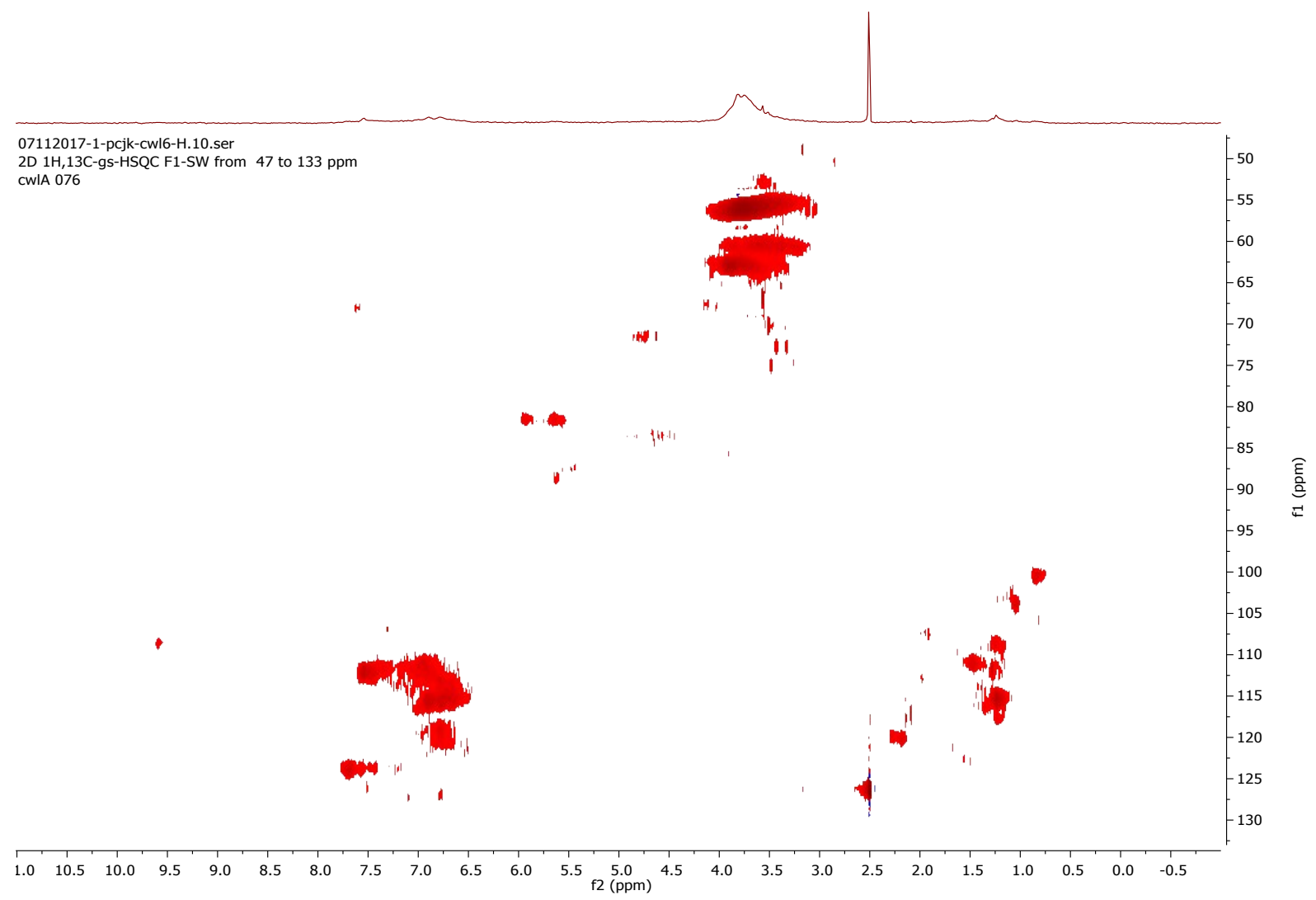

Figure S8 2D HSQC NMR spectrum of oxidised Douglas fir dioxosolv lignin in DMSO- $d_{6}$ obtained using $1 \mathrm{wt}$. eq. DDQ at room temperature for $24 \mathrm{hrs}$ 
Douglas fir lignin - 2 eq. DDQ, room temp

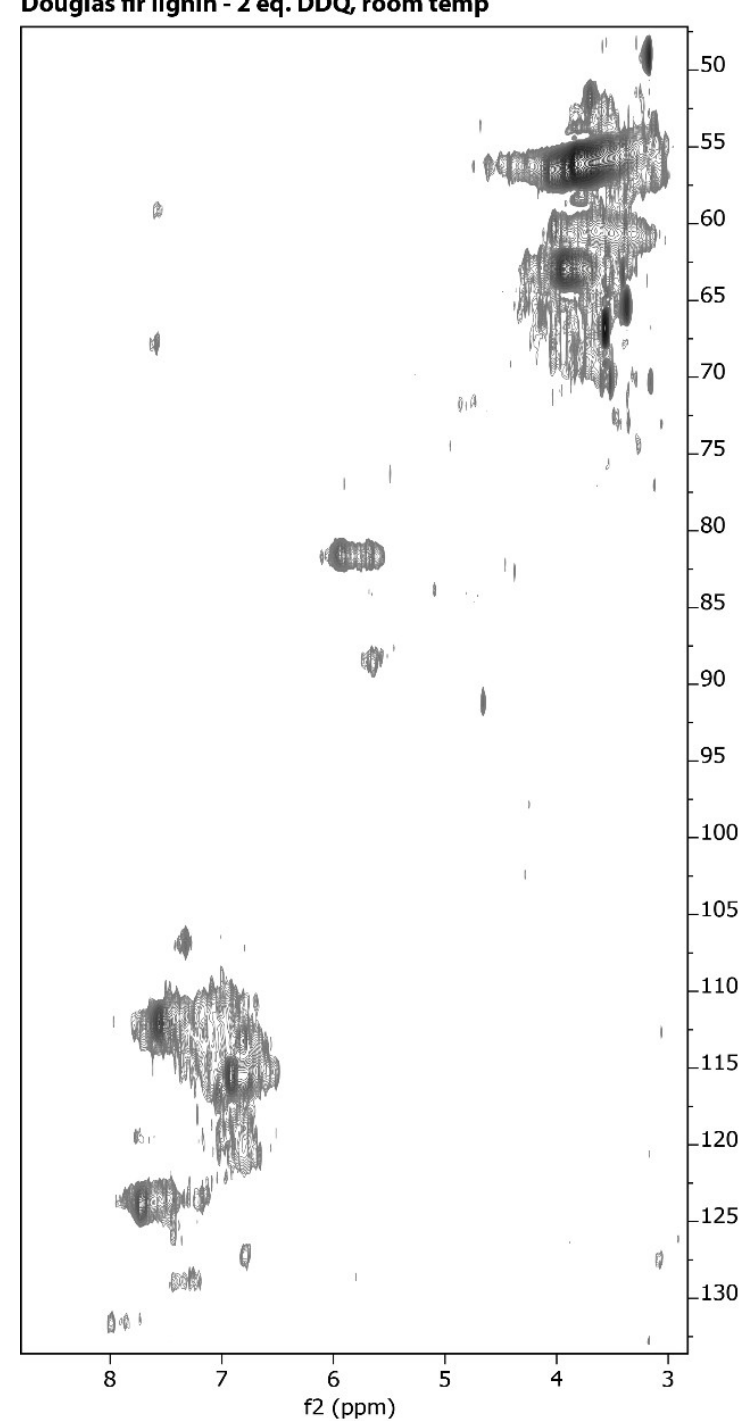

Compound 13

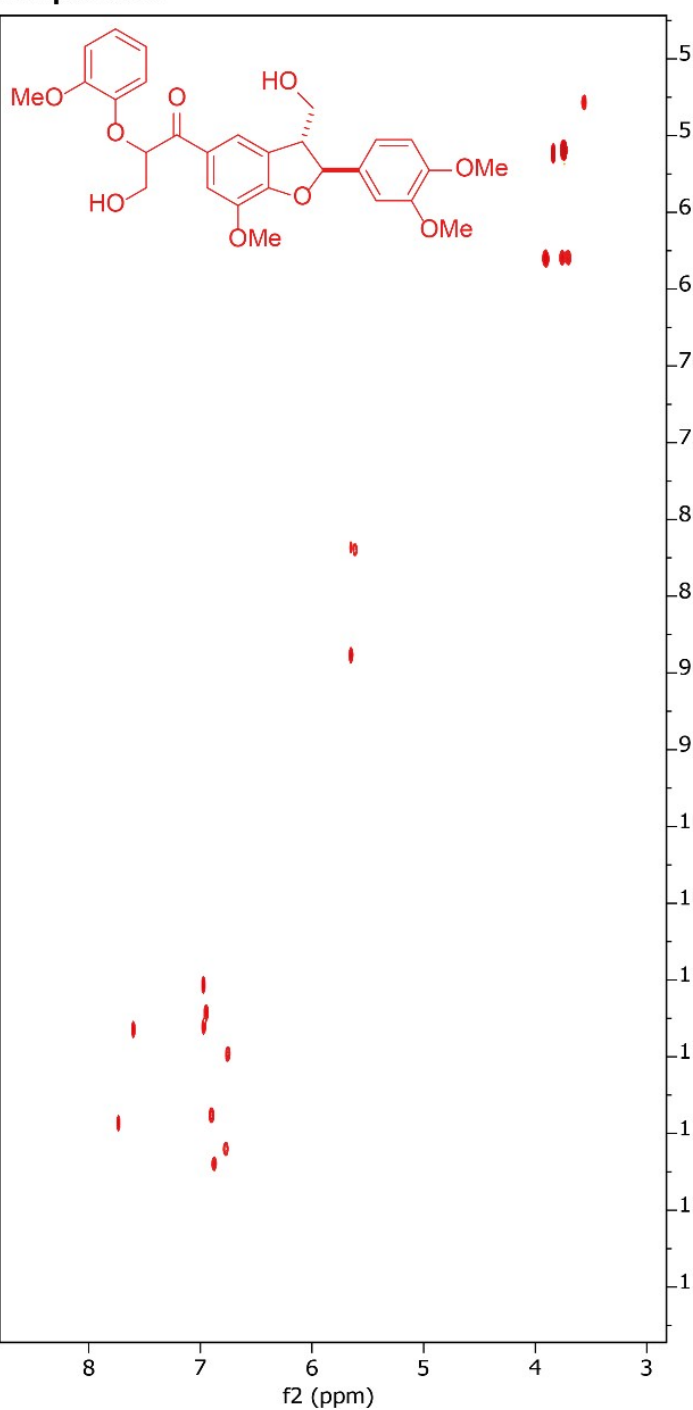

Overlay

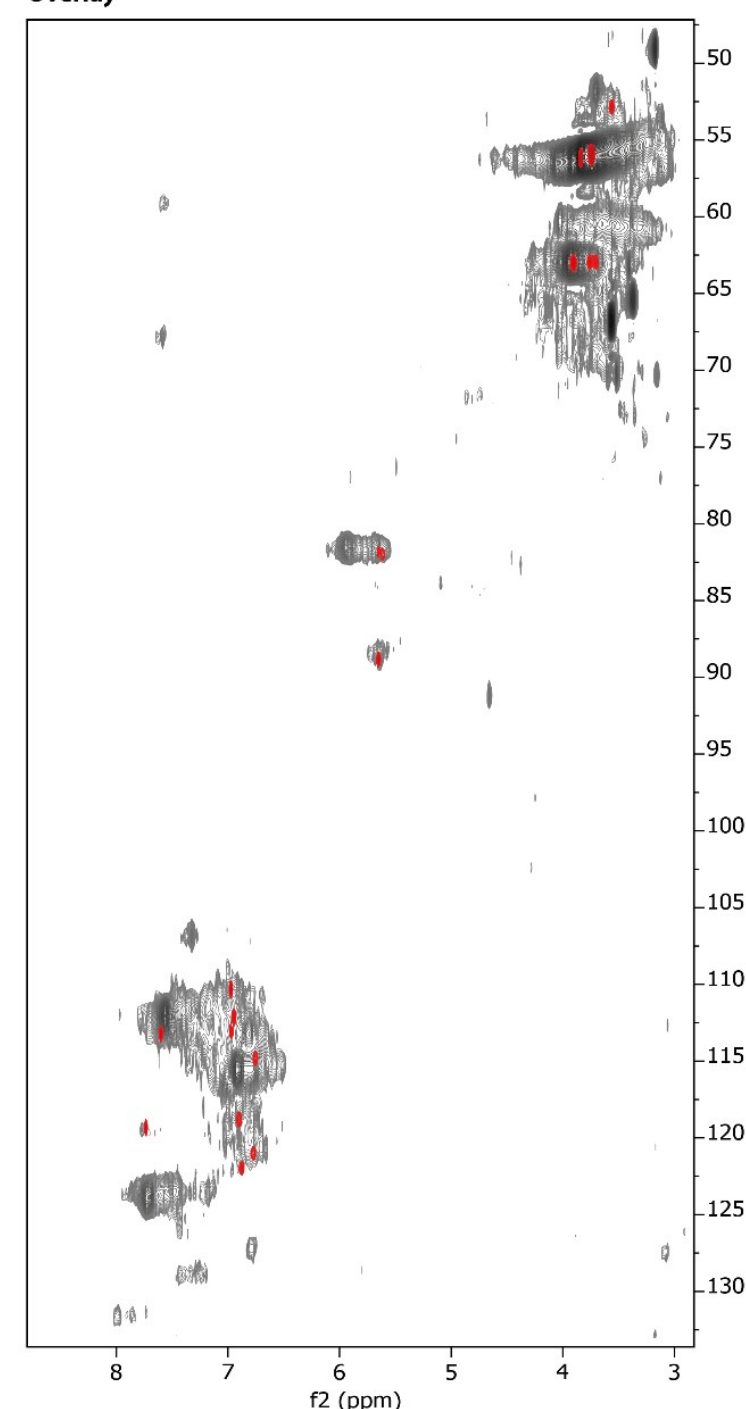

Figure S9 Comparison of model compound $\mathbf{1 3}$ to room temperature oxidised Douglas fir lignin. Characteristic cross peaks for the assignment of the $\beta-5$ unit next to an oxidised $\beta-\mathrm{O}-4$ unit are seen at $c a$. 5.6/88.4 ppm and 7.7/119 ppm in the model and lignin spectra. 

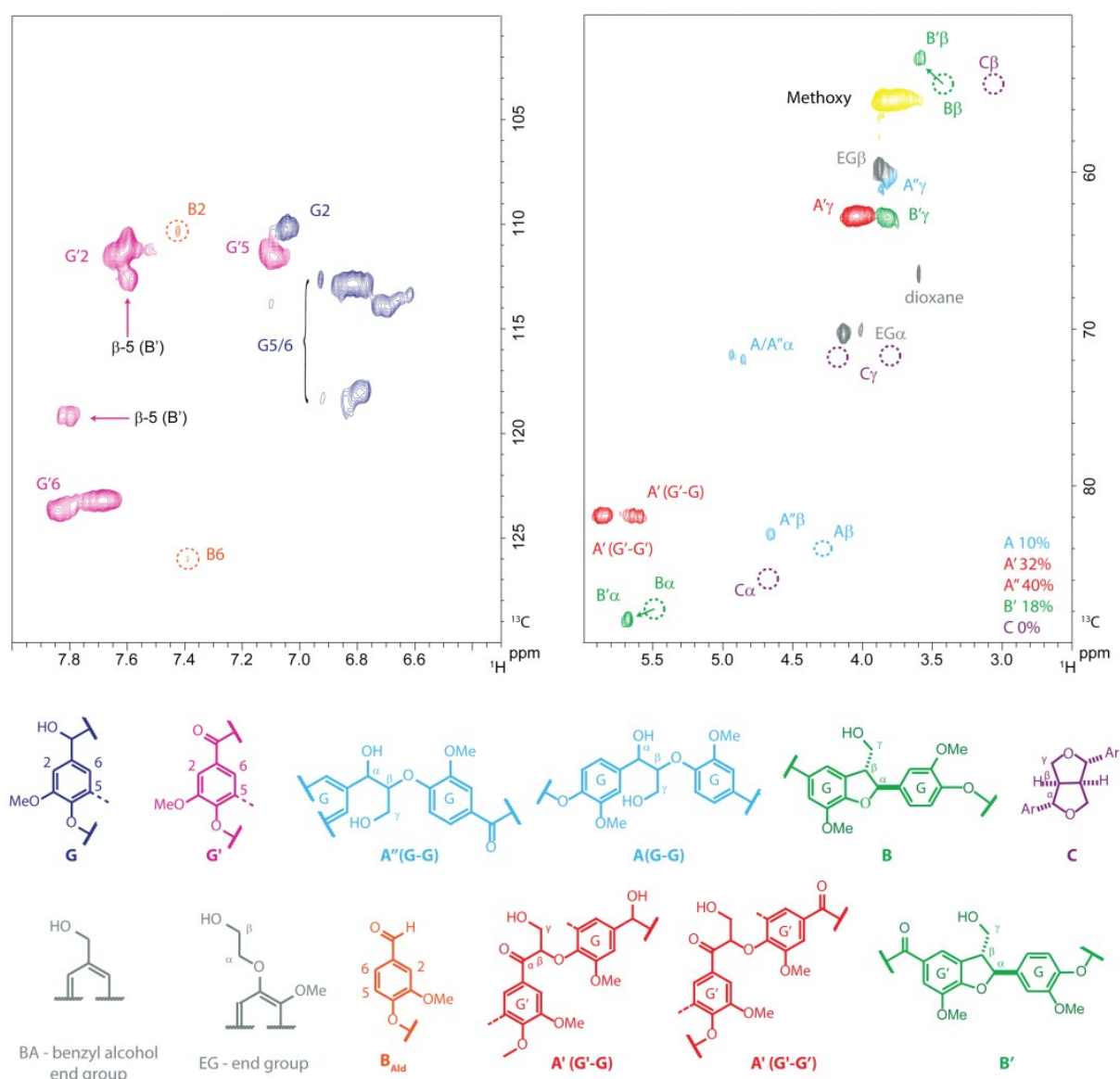

Figure S10: 2D HSQC NMR analysis of a DDQ oxidised model softwood lignin polymer (starting ratios A:B:C $=74: 17: 9$ ). Reagents and conditions: $1 \mathrm{wt}$ eq. DDQ, 1,4-dioxane/MeOH (9:1), r.t, 16 h. NMR - $500 \mathrm{MHz}$, $\mathrm{d}_{6}$-acetone/ $\mathrm{D}_{2} \mathrm{O}$ (9:1). 


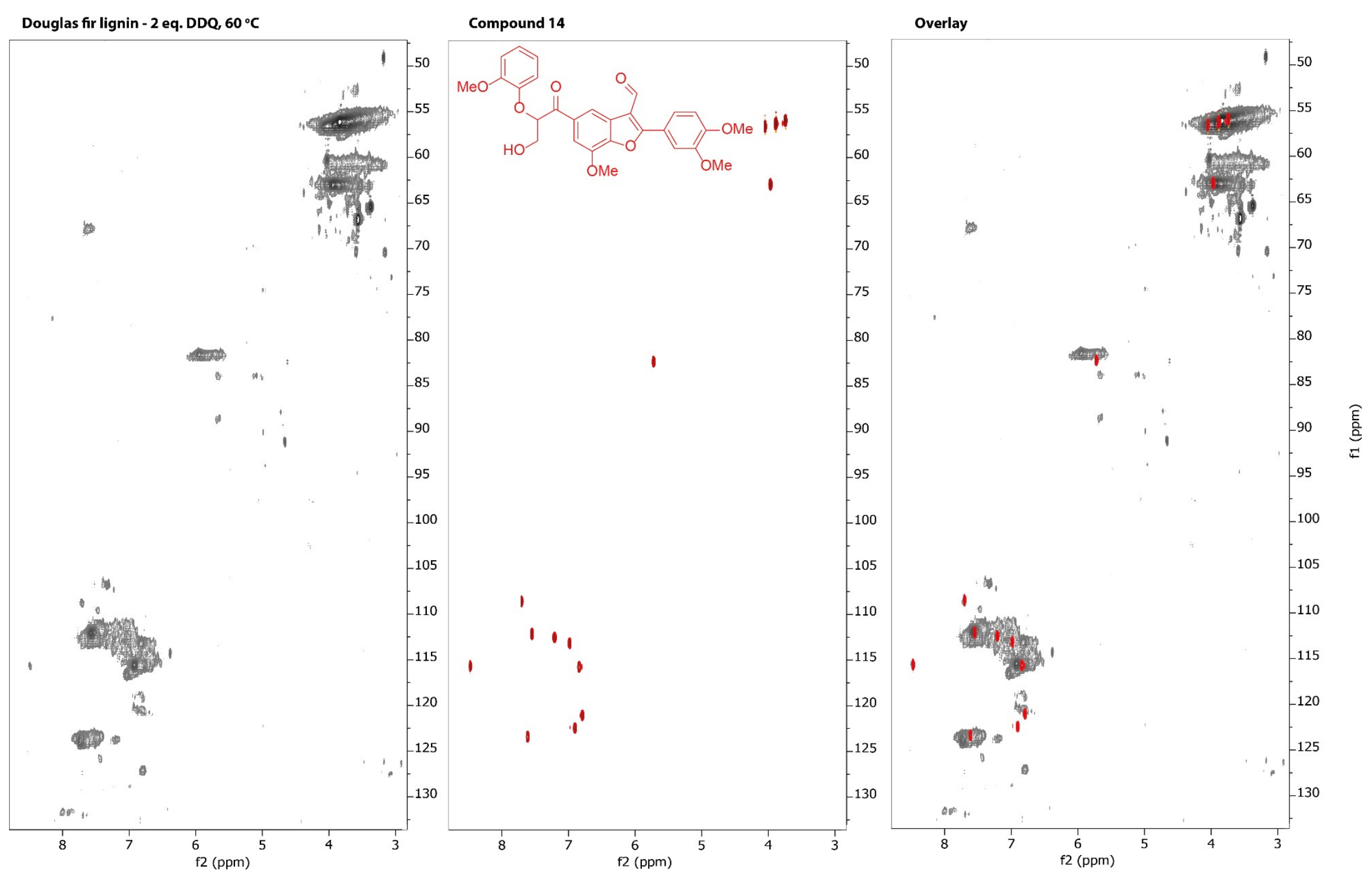

Figure S11 Comparison of model compound 14 to $60^{\circ} \mathrm{C}$ oxidised Douglas fir lignin. Characteristic cross peaks for the assignment of the oxidised $\beta-5$ unit are seen at $c a .7 .7 / 108 \mathrm{ppm}$ and 8.5/116 ppm in the model and lignin spectra. 


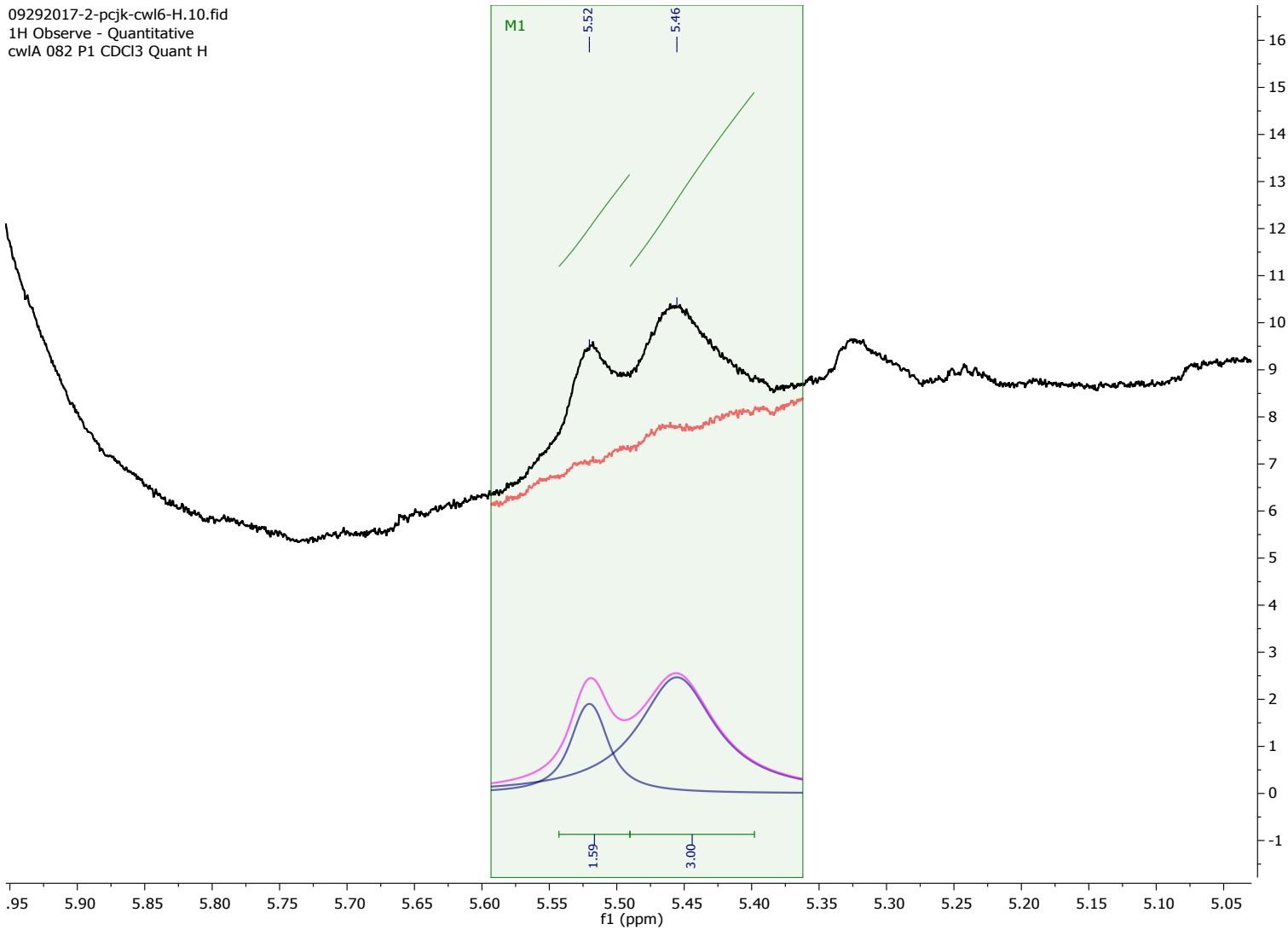

Figure S12: Quantitative ${ }^{1} \mathrm{H}$ NMR of acetylated Douglas fir dioxasolv lignin in $\mathrm{CDCl}_{3}(\mathrm{D} 1=30 \mathrm{~s})$ showing the integrations of the peaks corresponding to the phenolic $(5.52 \mathrm{ppm})$ and etherified $(5.46$ ppm) $\beta-5$ linkages. Deconvolution of these peaks in MestReNova (above) gave peak areas of 1:3 for the phenolic and non-phenolic peaks respectively. 


\section{$\underline{\text { S7.0 References }}$}

(1) Tran, F.; Lancefield, C. S.; Kamer, P. C. J.; Lebl, T.; Westwood, N. J. Green Chem. 2015, 17, 244.

(2) Pieters, L.; Van Dyck, S.; Gao, M.; Bai, R.; Hamel, E.; Vlietinck, A.; Lemière, G. J. Med. Chem. 1999, 42 (26), 5475.

(3) Lahive, C. W.; Deuss, P. J.; Lancefield, C. S.; Sun, Z.; Cordes, D. B.; Young, C. M.; Tran, F.; Slawin, A. M. Z.; de Vries, J. G.; Kamer, P. C. J.; Westwood, N. J.; Barta, K. J. Am. Chem. Soc. 2016, 138 (28), 8900.

(4) Nabeta, K.; Hirata, M.; Ohki, Y.; Samaraweera, S. W. A.; Okuyama, H. Phytochemistry 1994, 37 (2), 409.

(5) Ede, R. M.; Ralph, J. Magn. Reson. Chem. 1996, 34 (4), 261.

(6) Yang, Z.; Hon, P. M.; Chui, K. Y.; Xu, Z. Le; Chang, H. M.; Lee, C. M.; Cui, Y. X.; Wong, H. N.; Poon, C. D.; Fung, B. M. Tetrahedron Lett. 1991, 32 (18), 2061.

(7) Wang, E. C.; Wein, Y. S.; Kuo, Y. H. Tetrahedron Lett. 2006, 47 (52), 9195.

(8) Wang, Q.; Ding, M.; Jing, M. Hecheng Huaxue 2006, 14 (2), 162.

(9) Lancefield, C. S.; Westwood, N. J. Green Chem. 2015, 17 (11), 4980. 


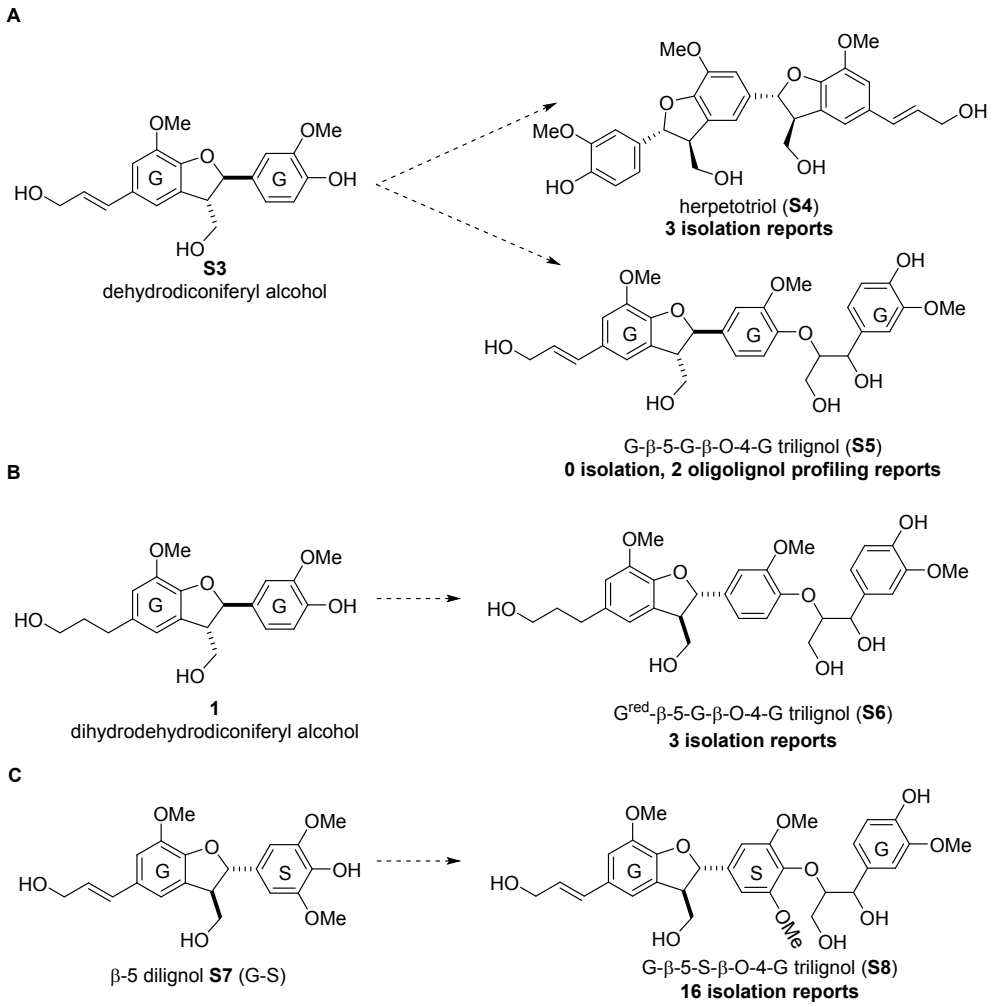

Figure S14 Natural $\beta-5$ trilignols or lignans coupled through the phenolic position. The relative lack of trilignols such as $\mathbf{S 5}$ and $\mathbf{S 6}$ indicates that the natural oxidative coupling of the $\beta-5$ dilignols $\mathbf{S 3}$ and/or 1 may be difficult. Data from the SciFinder database - November 2017. Different diastereomers of S5, $\mathbf{S 6}$ and $\mathbf{S 8}$ were grouped for counting purposes. S5 was identified in mass spectrometry based profiling of oligolignol from poplar xylem extracts.
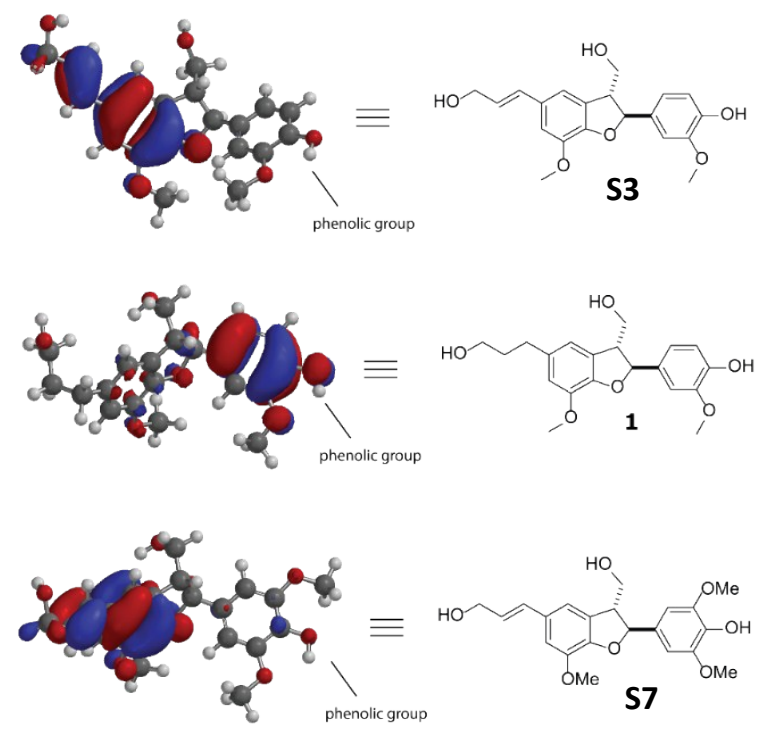

Figure S13: HOMO electron density maps of dilignol S3, the saturated derivative $\mathbf{1}$ and dilignol S7. Images are plotted at IsoValue $=0.032$. No orbital density is seen on the phenolic oxygen in S1, however a small amount can be seen in $\mathbf{S 7}$ and much more in $\mathbf{1}$. For each compound the lowest energy conformer identified from a conformer search, using the Merck Molecular Force Field (MMFF), was optimised at the AM1 level of theory and the HOMO density maps calculated at the HF/STO-3G level of theory. Calculations were carried out in the Spartan software package. 
We found from surveying lignan type natural products and available data from profiling of oligolignols in planta ${ }^{31,32}$ that oxidative coupling of dihydrodehydrodiconiferyl alcohol $\mathbf{1}$ and dehydrodiconiferyl alcohol $\mathbf{S 3}$ appears to be possible but perhaps quite challenging, whilst closely related $\beta-5$ dilignols such as S7, in which the terminal phenolic unit is a syringyl rather than guaiacyl unit, appear to cross couple quite readily. This was qualitatively judged by the relative numbers of natural products identified which have likely arisen from oxidative coupling of each substrate with coniferyl alcohol (Figure S13). Additionally, preliminary electron density calculations (See Figure S14) suggest that the allyl group in $\mathbf{S 3}$ dominates the HOMO in this dilignol, consistent with previous reports. ${ }^{29}$ However this effect is less apparent in $\mathbf{S 7}$ and does not exist in 1, with more electron orbital density being present on the phenolic groups in $\mathbf{S 7}$ and $\mathbf{1}$ compared to $\mathbf{S 3}$, as needed for oxidation by peroxidases and laccases.

S4: Isolations ${ }^{1-3}$ MS Identifications or other ${ }^{4}$

S5: MS Identifications or other ${ }^{4,5}$

S6: Isolations ${ }^{6-8}$

S8: Isolations $s^{9-24}$

References for trilignol identifications:

1. D'abrosca, B. et al. Isolation and characterization of new lignans from the leaves of Cestrum parqui. Nat. Prod. Res. 20, 293-298 (2006).

2. Fiorentino, A. et al. Lignans, neolignans and sesquilignans from Cestrum parqui l'Her. Biochem. Syst. Ecol. 35, 392-396 (2007).

3. Favre-Bonvin, J., Kaouadji, M. \& Mariotte, A. M. Structure de l'herpetotriol, sesquilignane trimere de l'alcool coniferylique, isole d'herpetospermum caudigerum wall. Tetrahedron Lett. 19, 4111-4112 (1978).

4. Araujo, P. et al. Mass spectrometry imaging: An expeditious and powerful technique for fast in situ lignin assessment in Eucalyptus. Anal. Chem. 86, 3415-3419 (2014).

5. Whetten, R. et al. Lignin Biosynthesis. Plant Cell 7, 1001-1013 (1995).

6. Masuoka, C., ITol, Y. \& Nohara, T. Antioxidative Constituents from Viticis trifoliae Fructus (Fruit of Vitex. Food Sci. Technol. Int. Toky'o 4, 9-13 (1998).

7. Jiang, H. et al. New phenylpropanoid derivatives from the fruits of Xanthium sibiricum and their anti-inflammatory activity. Fitoterapia 117, 11-15 (2017).

8. Miki, K., Sasaya, T. \& Sakakibara, A. Structures of new lignans from larix leptolepis gord. Tetrahedron Lett. 20, 799-802 (1979).

9. Bai, Y. et al. Bioactives from stems and leaves of mung beans (Vigna radiata L.). J. Funct. Foods 25, 314-322 (2016).

10. Zhongguo ke xue yuan. Chengdu fen yuan. \& Zhongguo ke xue yuan. Chengdu wen xian qing bao zhong xin. Tian ran chan wu yan jiu yu kai fa = Natural product R \&amp; D. Nat. Prod. Res. Dev.

11. Li, S. F. et al. Anthraquinones and lignans from Cassia occidentalis. Helv. Chim. Acta 93, 1795-1802 (2010).

12. Morreel, K. et al. Profiling of oligolignols reveals monolignol coupling conditions in lignifying poplar xylem. Plant Physiol. 136, 3537-49 (2004). 
13. Damiani, I. et al. Metabolite profiling reveals a role for atypical cinnamyl alcohol dehydrogenase CAD1 in the synthesis of coniferyl alcohol in tobacco xylem. Plant Mol. Biol. 59, 753769 (2005).

14. Kim, N. H., Yang, M. H., Heo, J. D., Sung, S. H. \& Jeong, E. J. Dihydrobenzofuran neolignans isolated from Euonymus alatus leaves and twigs attenuated inflammatory responses in the activated RAW264.7 macrophage cells. Nat. Prod. Sci. 22, 53-59 (2016).

15. Jeong, E. J., Cho, J. H., Sung, S. H., Kim, S. Y. \& Kim, Y. C. Inhibition of nitric oxide production in lipopolysaccharide-stimulated RAW264.7 macrophage cells by lignans isolated from Euonymus alatus leaves and twigs. Bioorganic Med. Chem. Lett. 21, 2283-2286 (2011).

16. Li, X. et al. Antioxidant compounds from Rosa laevigata fruits. Food Chem. 130, 575-580 (2012).

17. Han, H. Y., Liu, H. W., Wang, N. L. \& Yao, X. S. Sesquilignans and dilignans from Campylotropis hirtella (Franch.) Schindl. Nat. Prod. Res. 22, 990-995 (2008).

18. Li, W. W. \& Barz, W. Structure and accumulation of phenolics in elicited Echinacea purpurea cell cultures. Planta Med. 72, 248-254 (2006).

19. Yoshikawa, K., Kageyama, H. \& Arihara, S. Phenolic glucosides and lignans from Ehretia ovalifolia. Phytochemistry 39, 659-664 (1995).

20. J. Houghton, P. Lignans and neolignans from Buddleja davidii. Phytochemistry 24, 819-826 (1985).

21. Sun, H. et al. Characterization of the multiple components of Acanthopanax Senticosus stem by ultra high performance liquid chromatography with quadrupole time-of-flight tandem mass spectrometry. J. Sep. Sci. 39, 496-502 (2016).

22. Cutillo, F., D'Abrosca, B., DellaGreca, M., Fiorentino, A. \& Zarrelli, A. Lignans and neolignans from Brassica fruticulosa: Effects on seed germination and plant growth. J. Agric. Food Chem. 51, 6165-6172 (2003).

23. Zhu, J.-Y. et al. Enantiomeric neolignans and sesquineolignans from Jatropha integerrima and their absolute configurations. RSC Adv. 5, 12202-12208 (2015).

24. Choi, G. et al. A Comparative Study on Hulled Adlay and Unhulled Adlay through Evaluation of Their LPS-Induced Anti-Inflammatory Effects, and Isolation of Pure Compounds. Chem. Biodivers. 12, 380-387 (2015). 


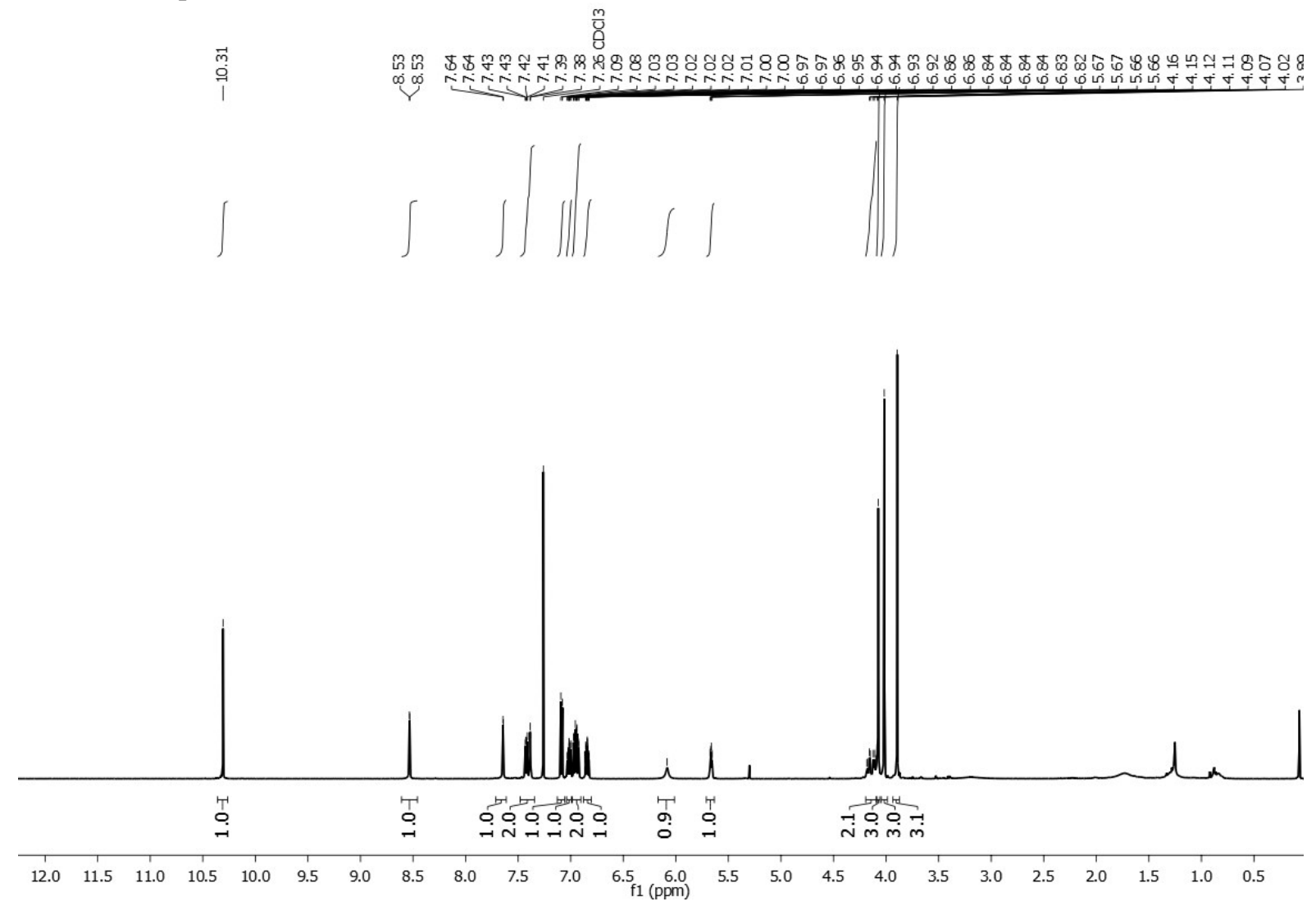

Compound 11: ${ }^{1} \mathrm{H} \mathrm{NMR}, 500 \mathrm{MHz}, \mathrm{CDCl}_{3}$

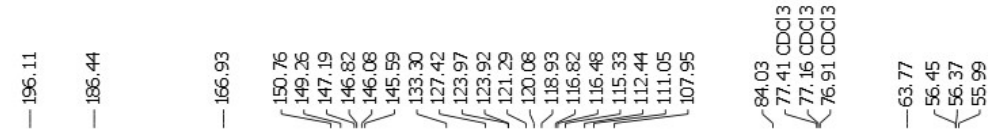

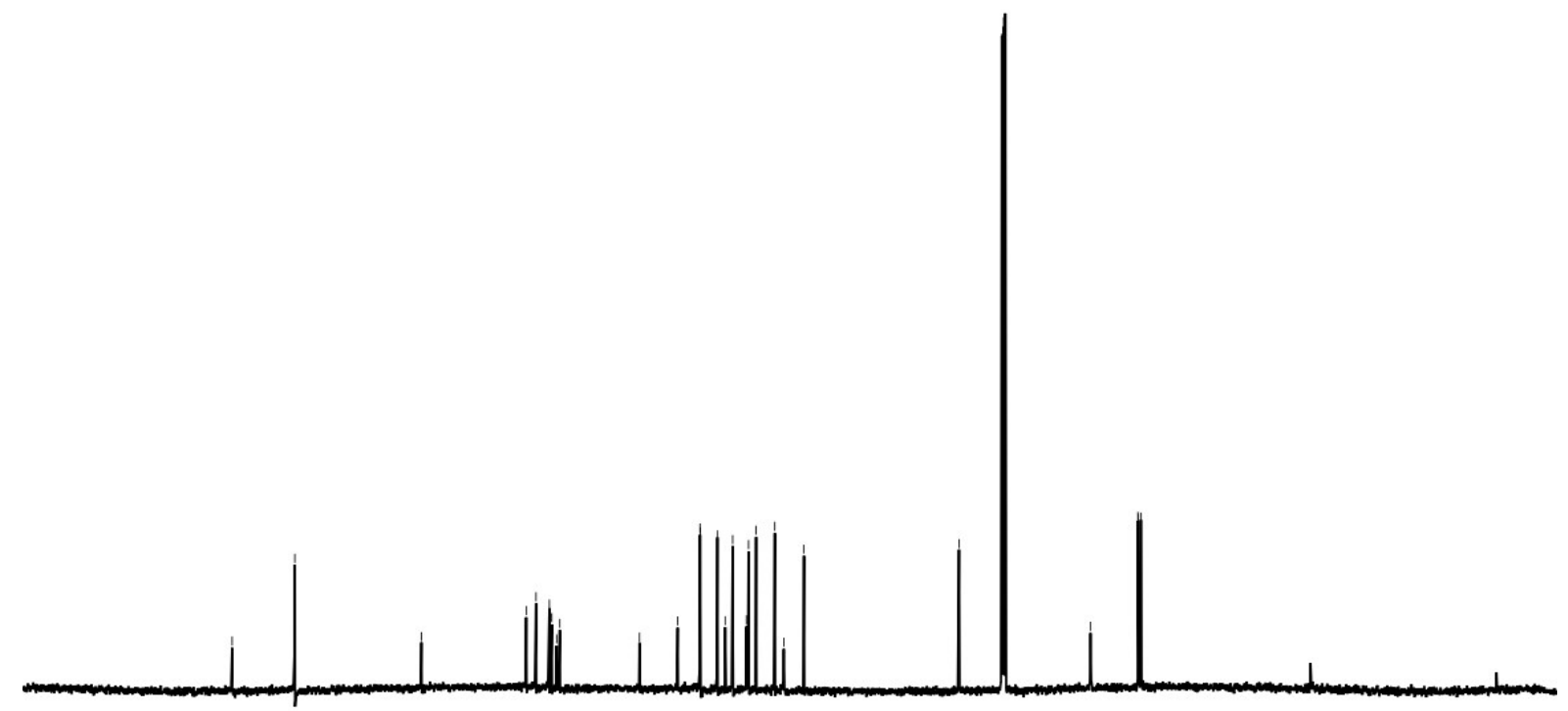

$\begin{array}{llllllllllllllllllllllll}220 & 210 & 200 & 190 & 180 & 170 & 160 & 150 & 140 & 130 & 120 & \begin{array}{c}110 \\ \mathrm{f} 1\end{array}(\mathrm{ppm}) & 100 & 90 & 80 & 70 & 60 & 50 & 40 & 30 & 20 & 10 & 0\end{array}$

Compound 11: ${ }^{13} \mathrm{C}$ NMR, $126 \mathrm{MHz}, \mathrm{CDCl}_{3}$ 

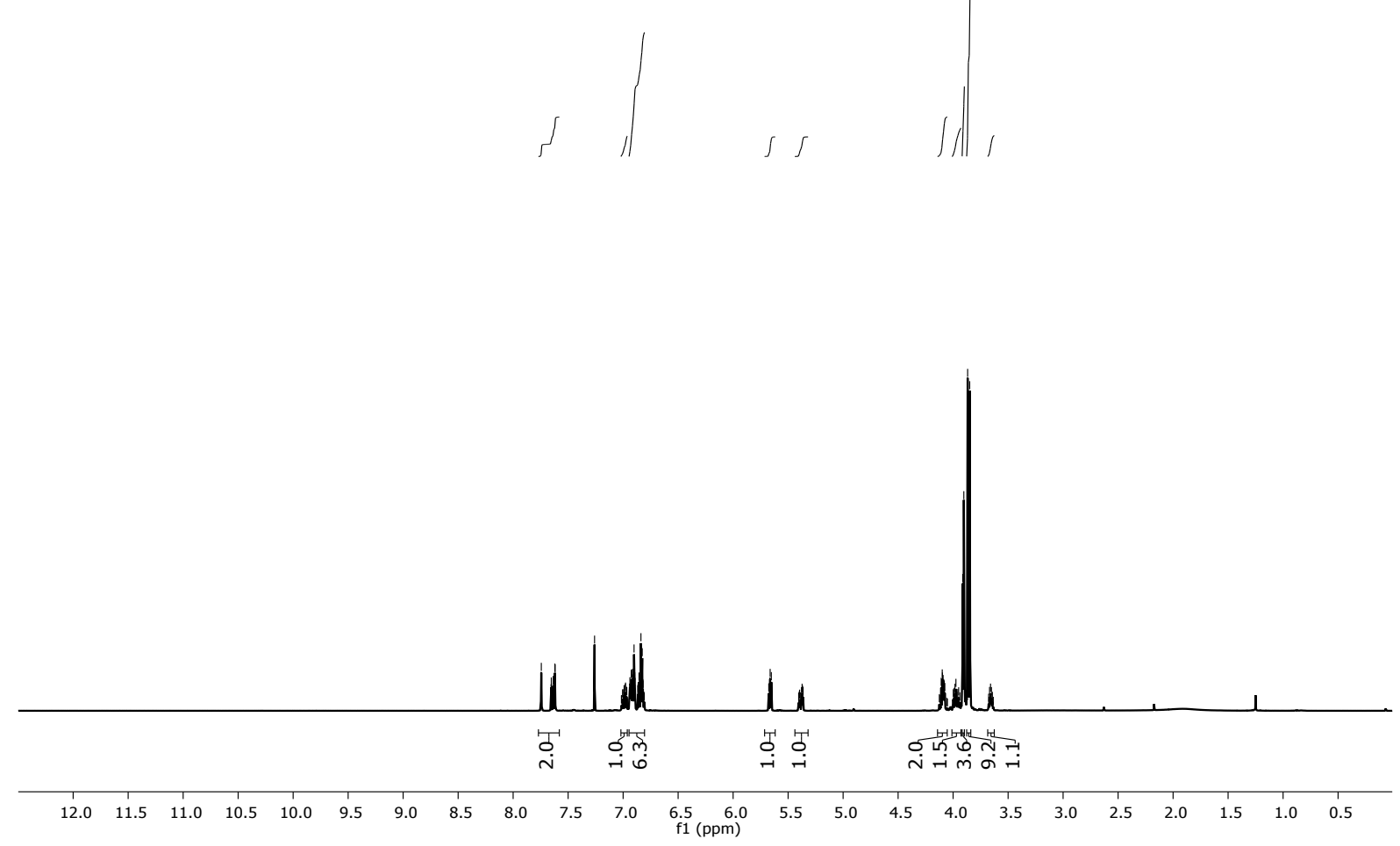

Compound 13: ${ }^{1} \mathrm{H} \mathrm{NMR}, 700 \mathrm{MHz}, \mathrm{CDCl}_{3}$

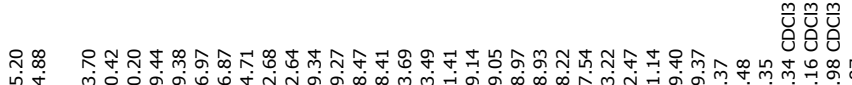

幽

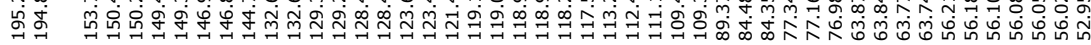

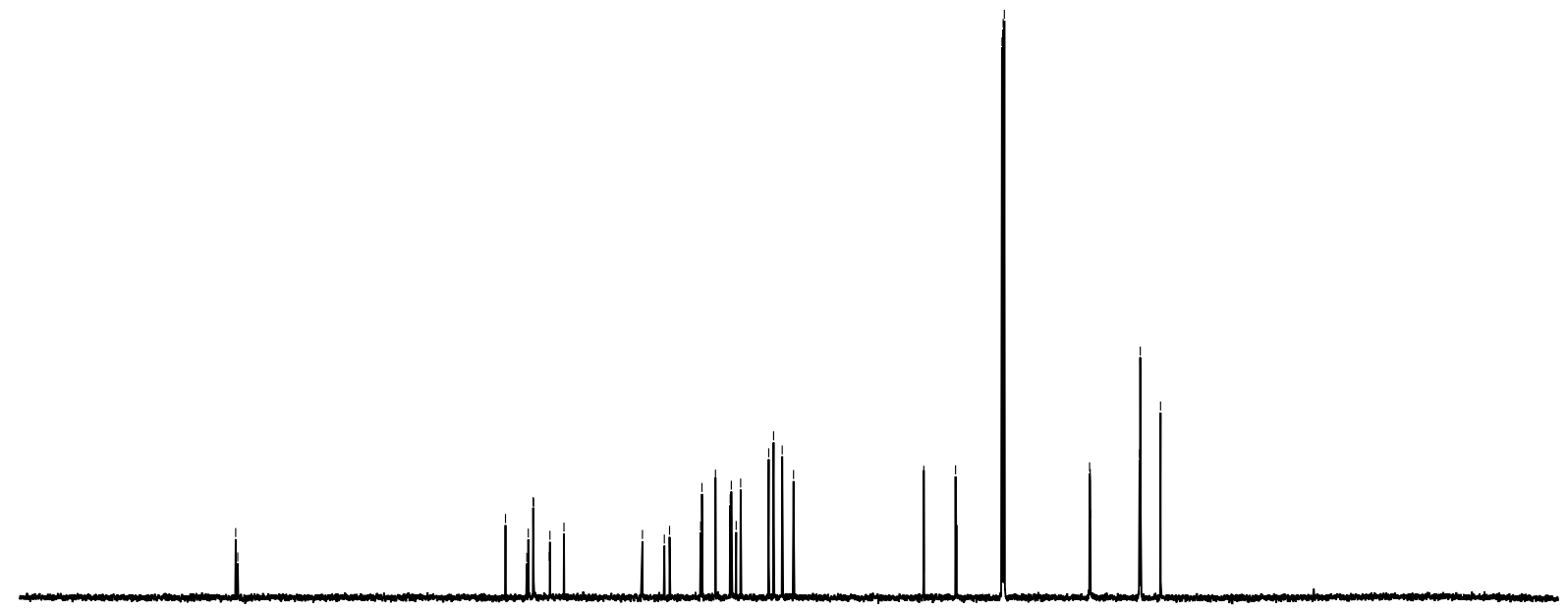

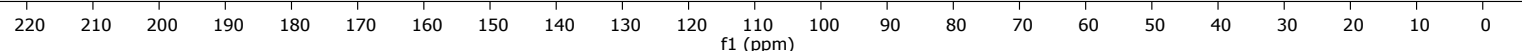

Compound 13: ${ }^{13} \mathrm{C}$ NMR, $176 \mathrm{MHz}, \mathrm{CDCl}_{3}$ 


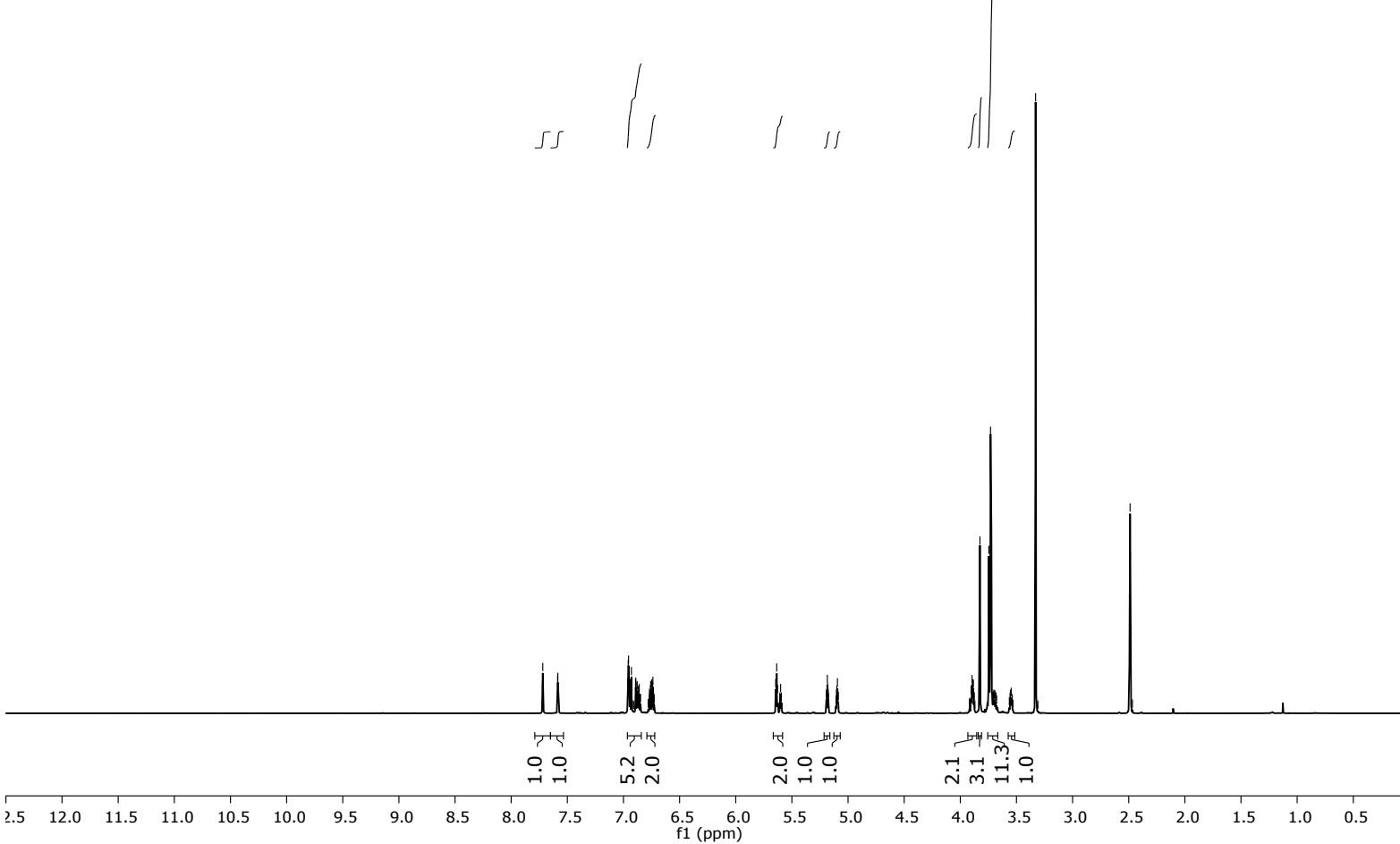

Compound 13: ${ }^{1} \mathrm{H}$ NMR, $700 \mathrm{MHz}$, DMSO- $d_{6}$

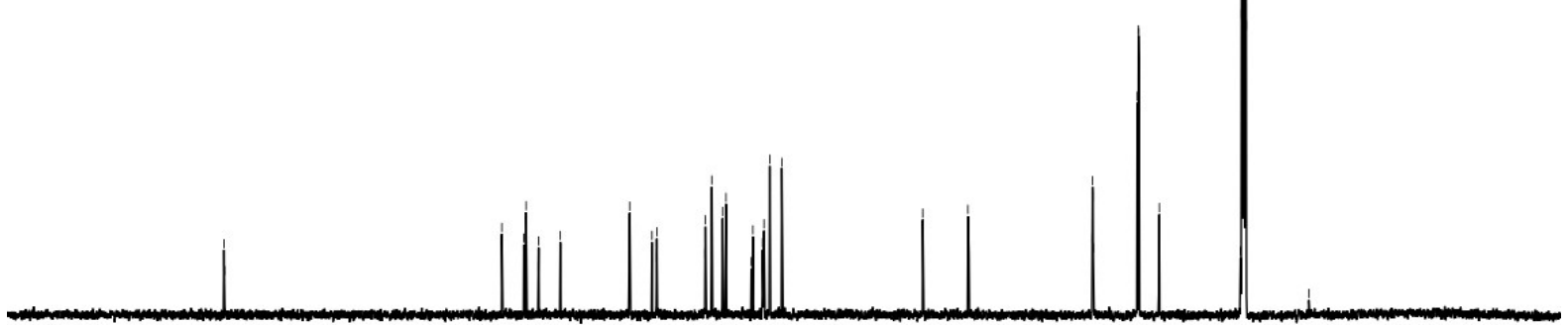

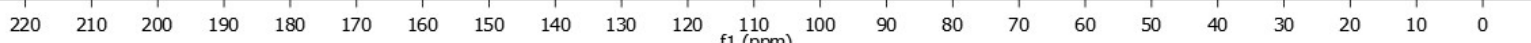

Compound 13: ${ }^{13} \mathrm{C}$ NMR, $176 \mathrm{MHz}$, DMSO- $d_{6}$ 

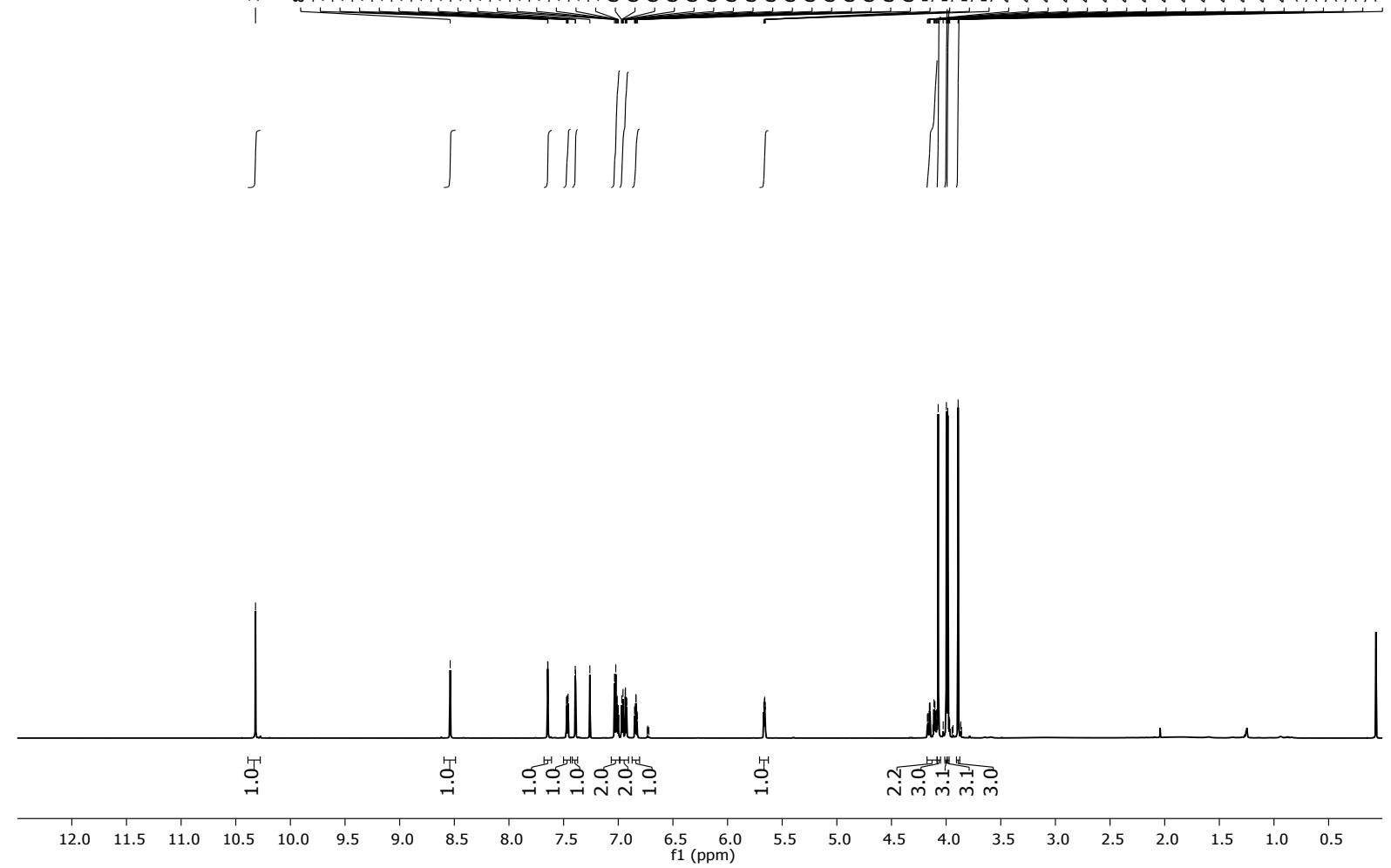

Compound 14: ${ }^{1} \mathrm{H} \mathrm{NMR}, 700 \mathrm{MHz}, \mathrm{CDCl}_{3}$

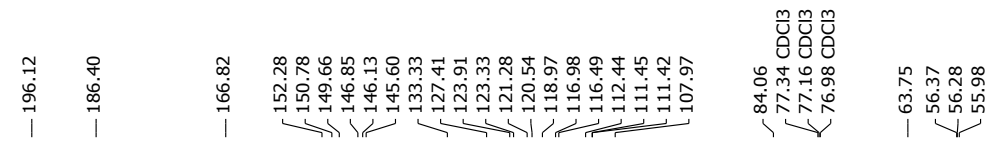

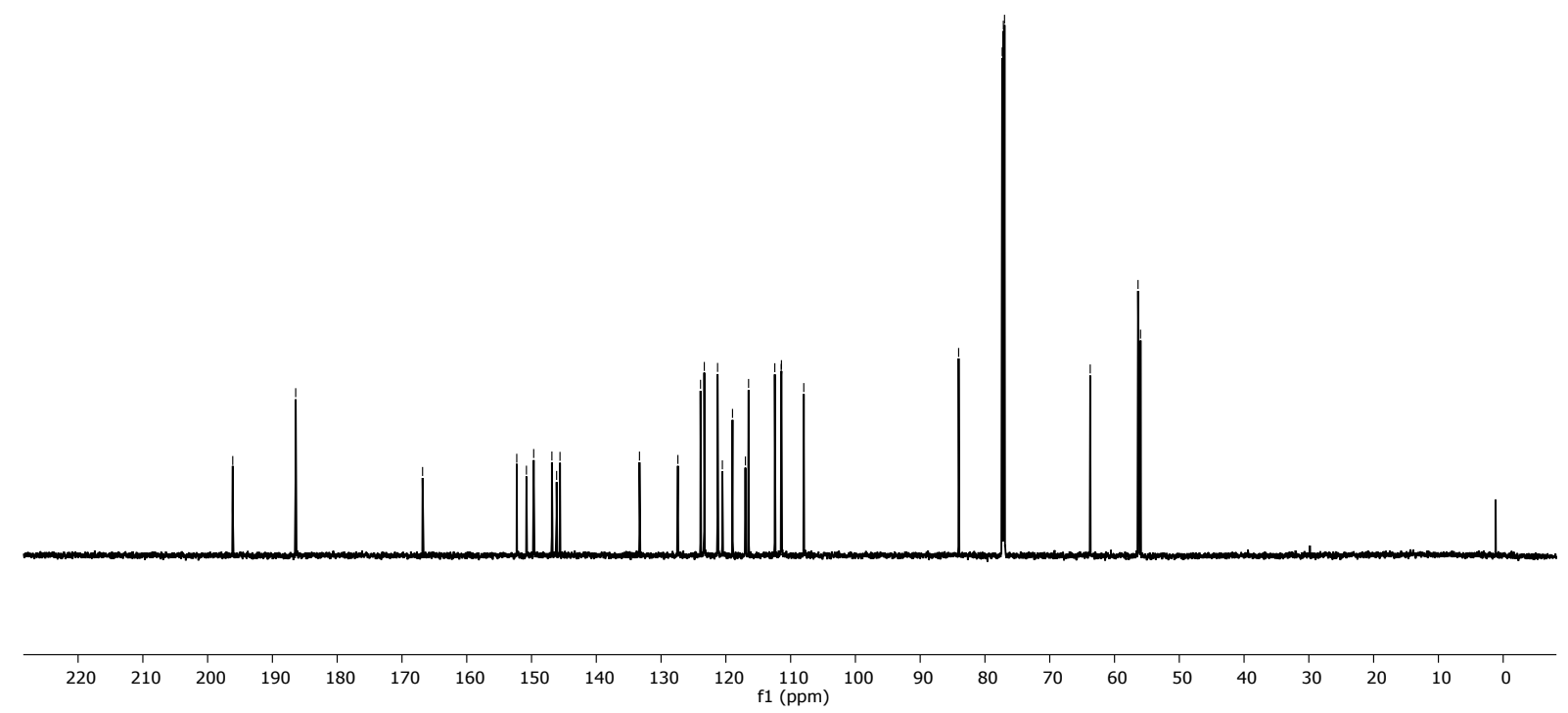

Compound 14: ${ }^{13} \mathrm{C}$ NMR, $176 \mathrm{MHz}, \mathrm{CDCl}_{3}$ 


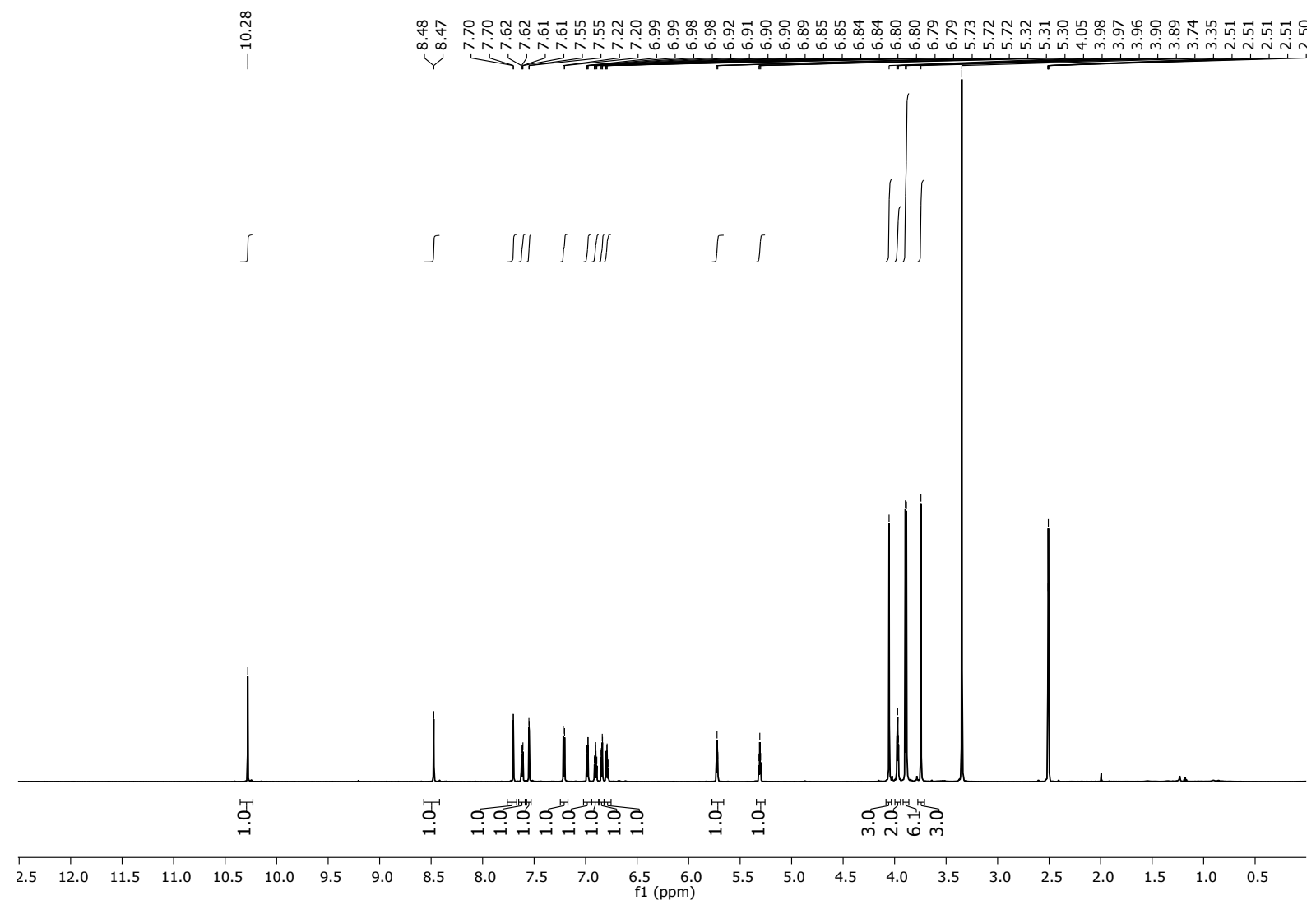

Compound 14: ${ }^{1} \mathrm{H}$ NMR, $700 \mathrm{MHz}$, DMSO- $d_{6}$
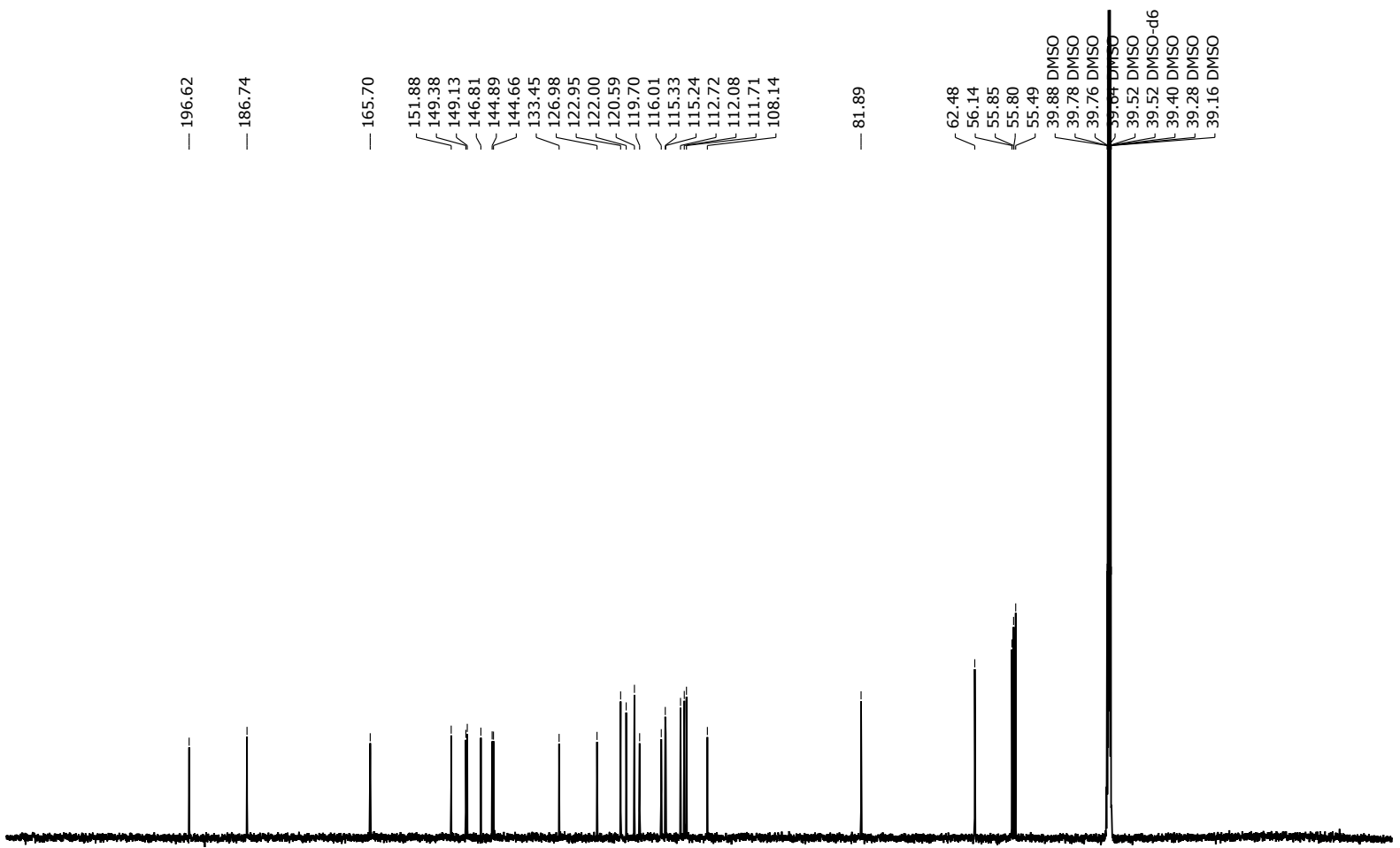

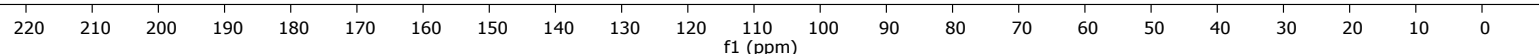

Compound 14: ${ }^{13} \mathrm{C}$ NMR, $176 \mathrm{MHz}$, DMSO- $d_{6}$ 


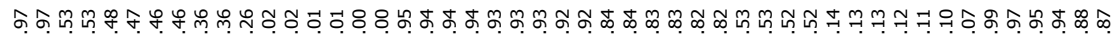

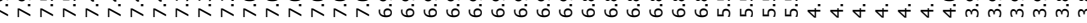
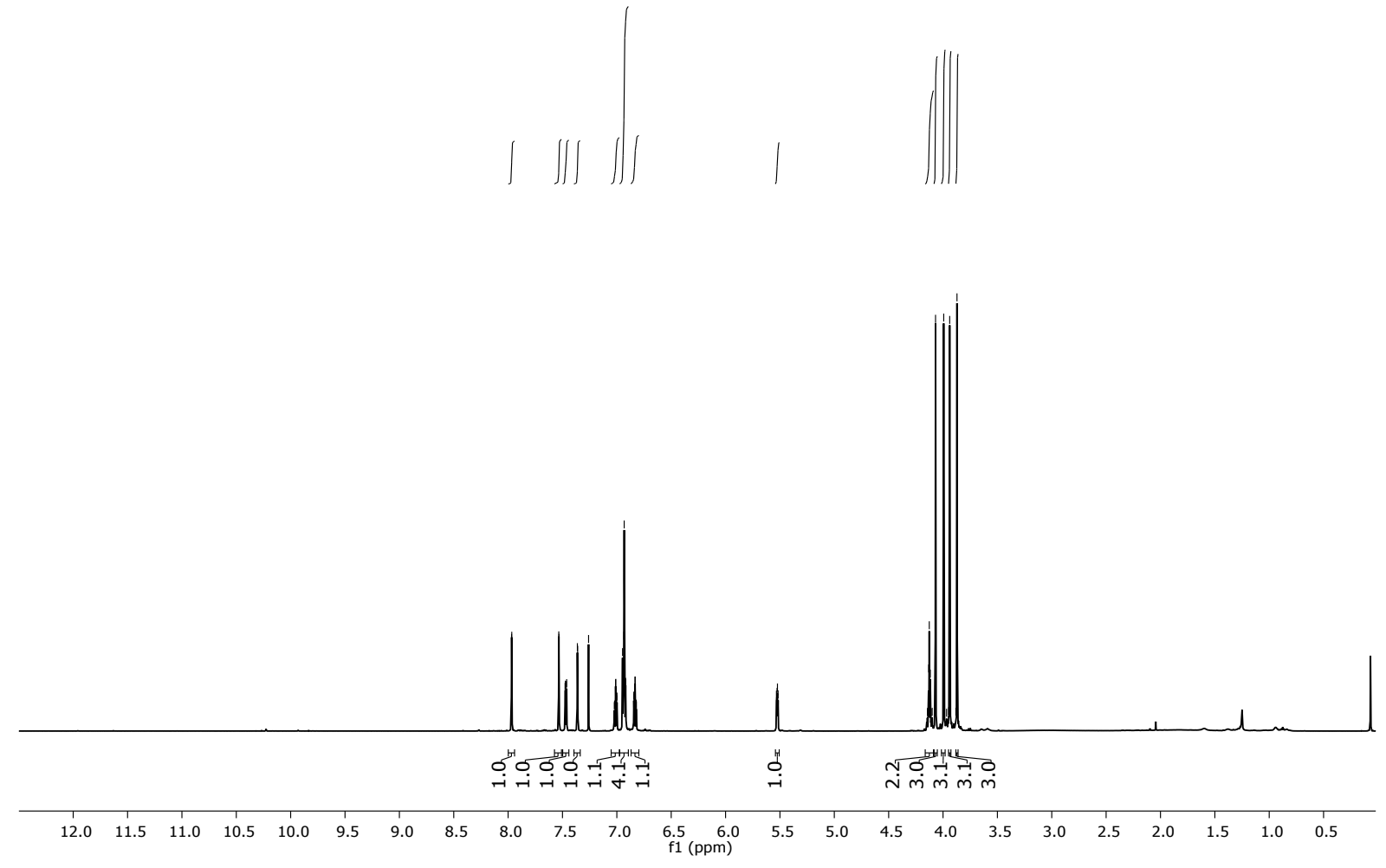

Compound 15: ${ }^{1} \mathrm{H} \mathrm{NMR}, 700 \mathrm{MHz}, \mathrm{CDCl}_{3}$

ลे

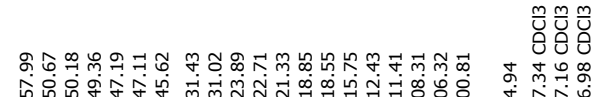

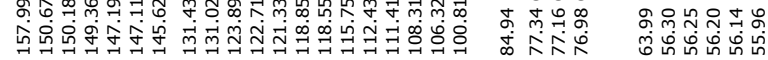
1 V

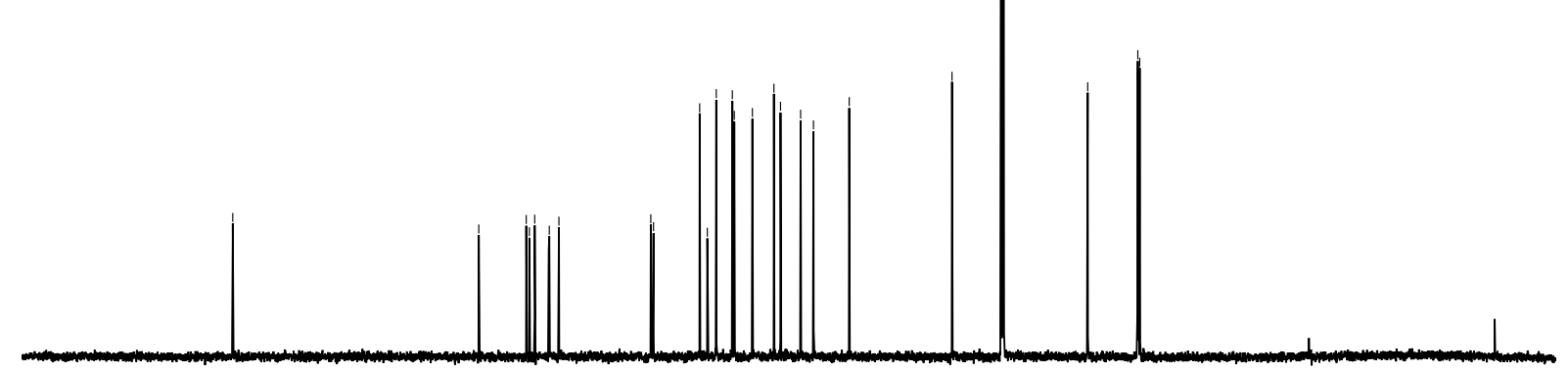

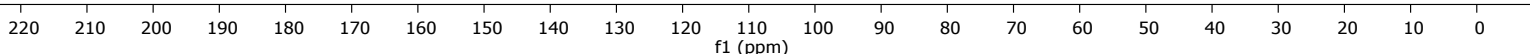
Compound 15: ${ }^{13} \mathrm{C} \mathrm{NMR,}, 176 \mathrm{MHz}, \mathrm{CDCl}_{3}$ 


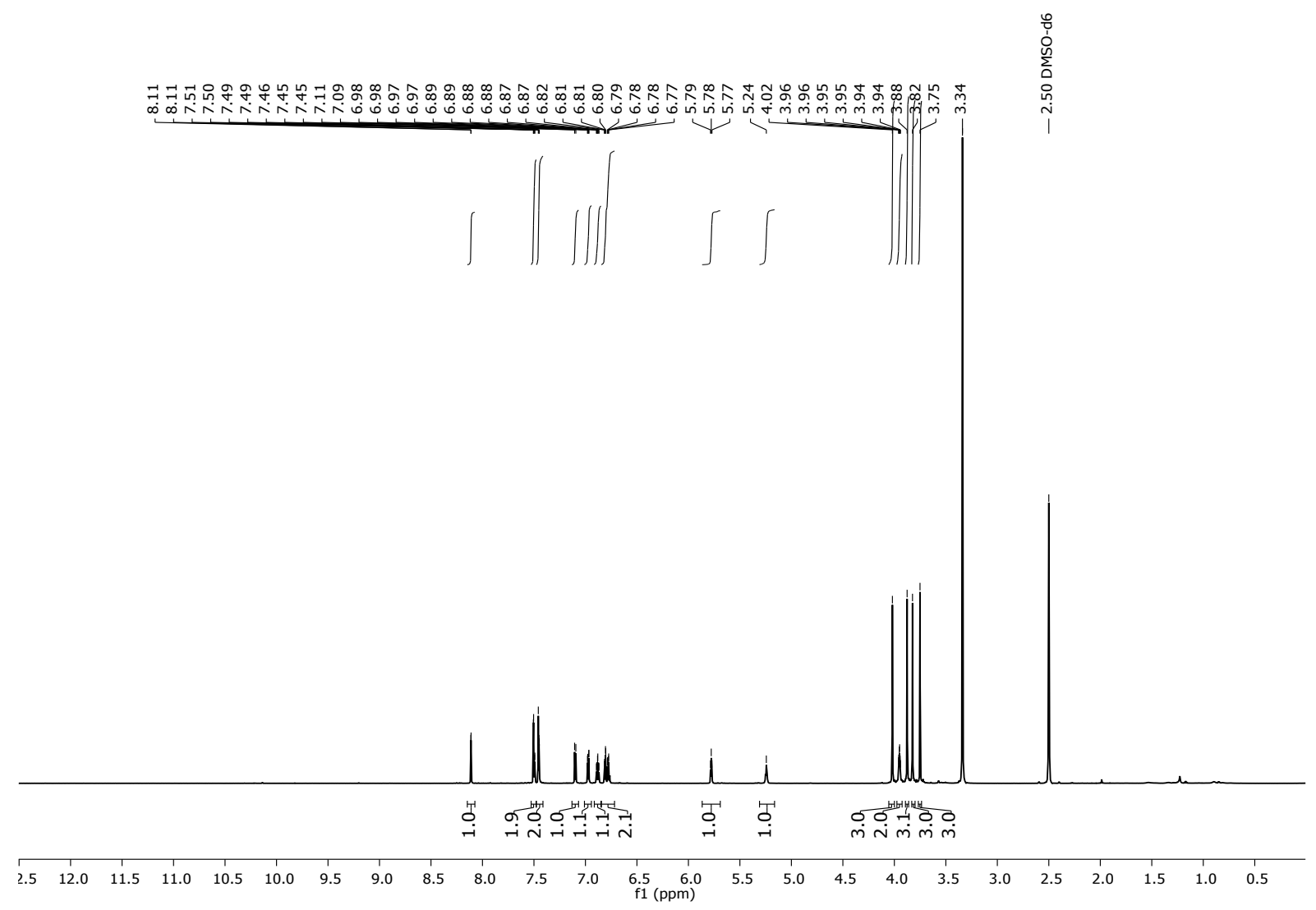

Compound 15: ${ }^{1} \mathrm{H}$ NMR, $700 \mathrm{MHz}$, DMSO- $d_{6}$

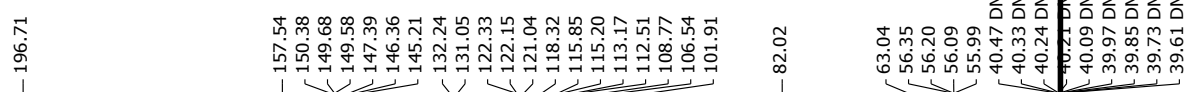
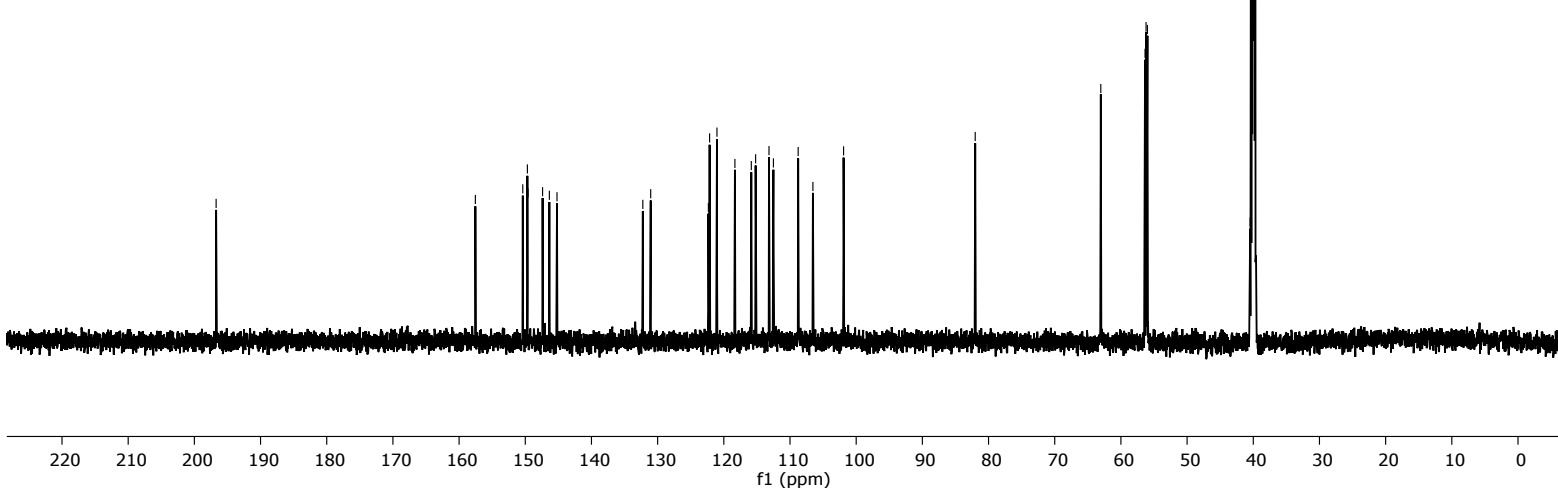

Compound 15: ${ }^{13} \mathrm{C}$ NMR, $176 \mathrm{MHz}$, DMSO- $d_{6}$ 


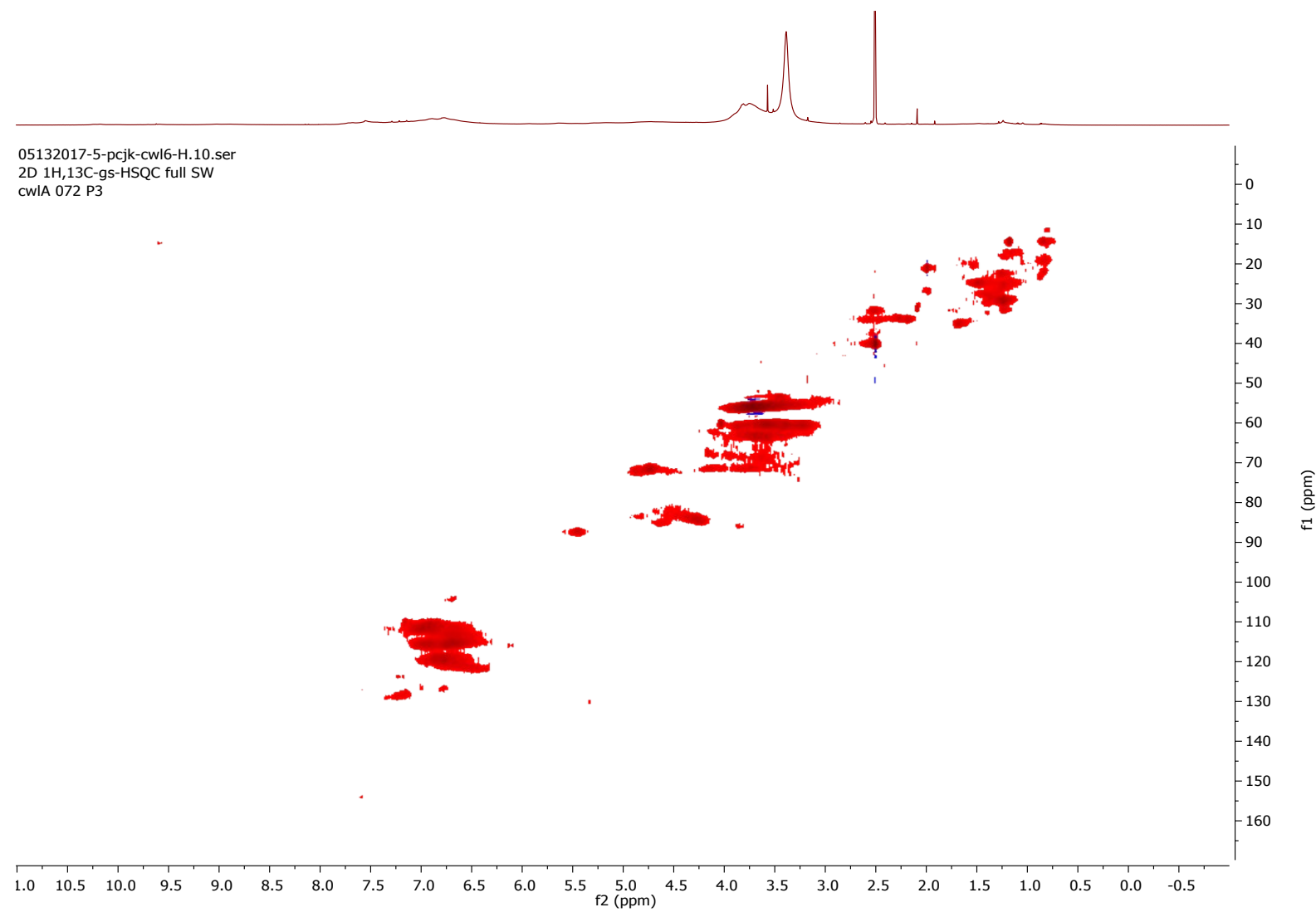

2D HSQC NMR of Douglas fir dioxosolv lignin in DMSO- $d_{6}$

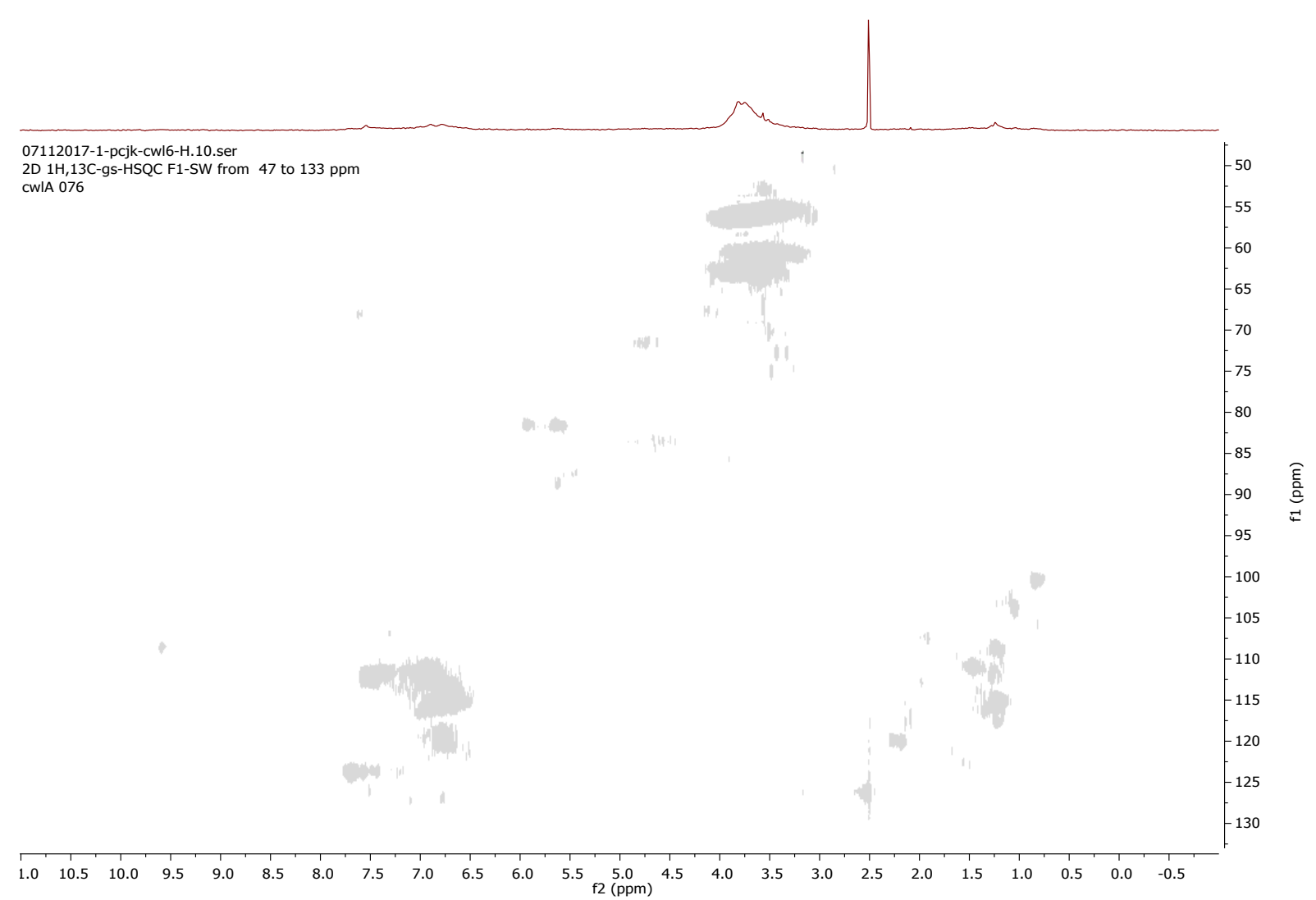

2D HSQC NMR of oxidised Douglas fir dioxosolv lignin in DMSO- $d_{6}$. Oxidised with 1 eq. DDQ at room temperature for $24 \mathrm{hrs}$ 


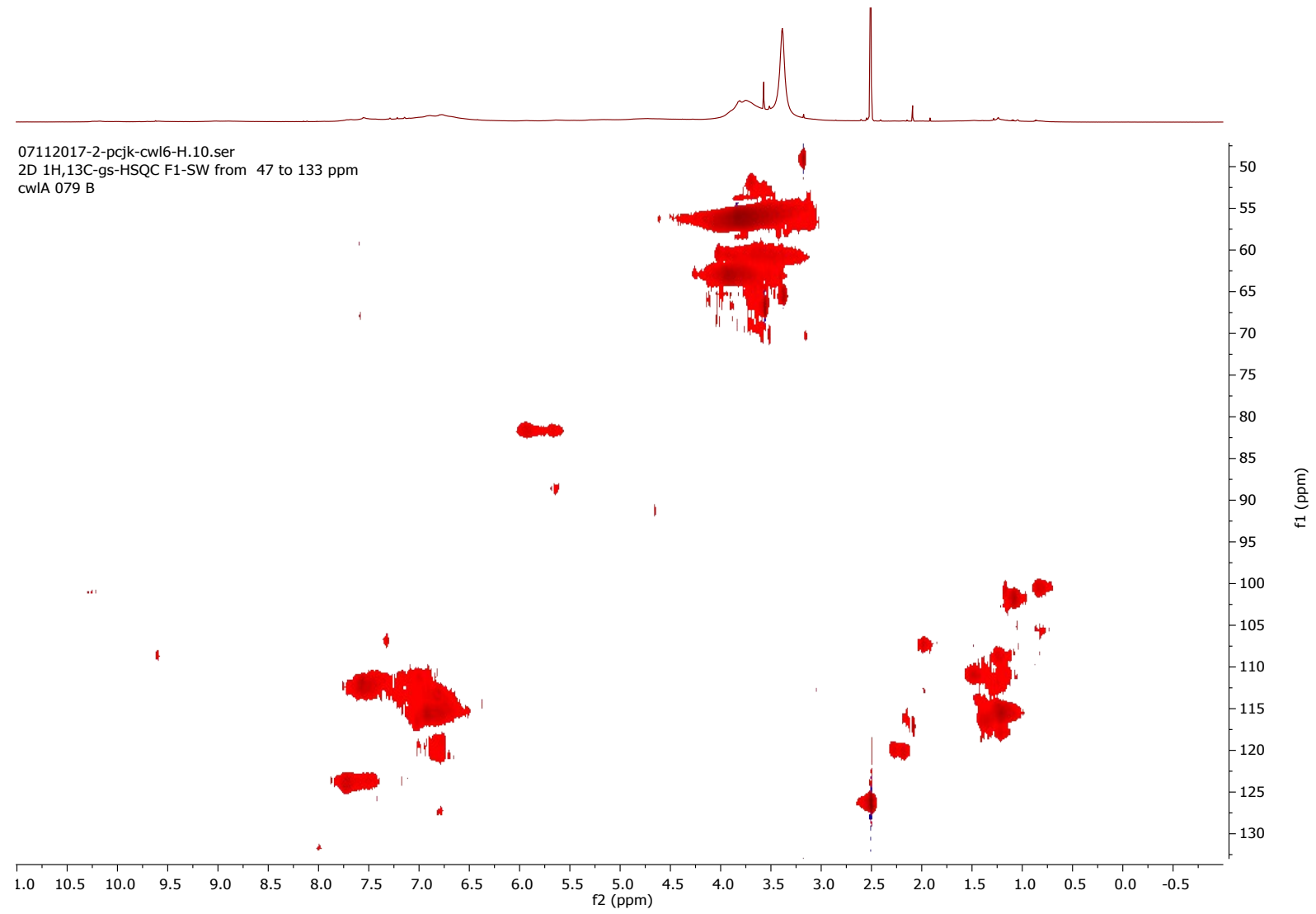

2D HSQC NMR of oxidised Douglas fir dioxosolv lignin in DMSO- $d_{6}$. Oxidised with 2 eq. DDQ at room temperature for $24 \mathrm{hrs}$

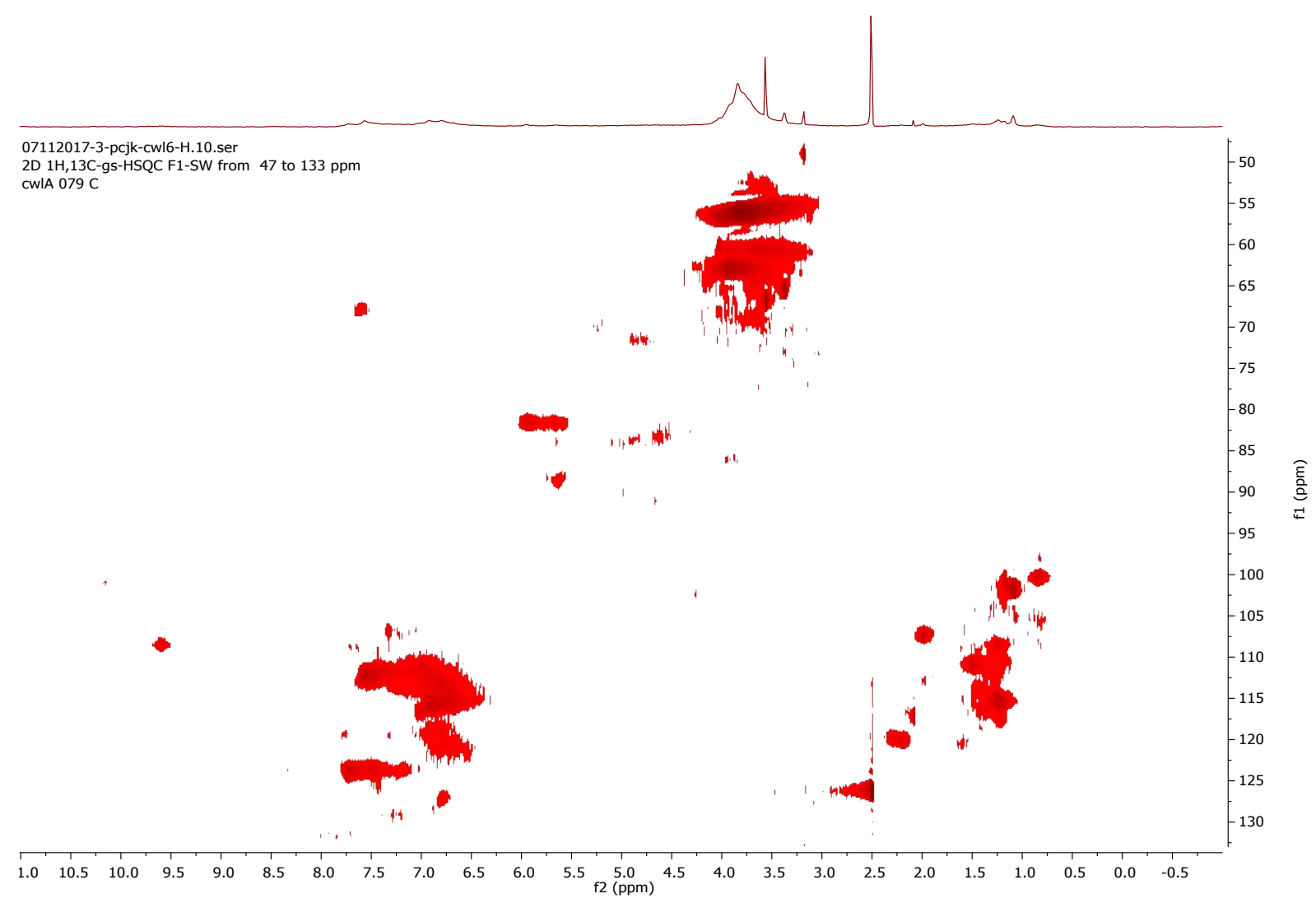

2D HSQC NMR of oxidised Douglas fir dioxosolv lignin in DMSO- $d_{6}$. Oxidised with 1 eq. DDQ at $60{ }^{\circ} \mathrm{C}$ for $24 \mathrm{hrs}$ 


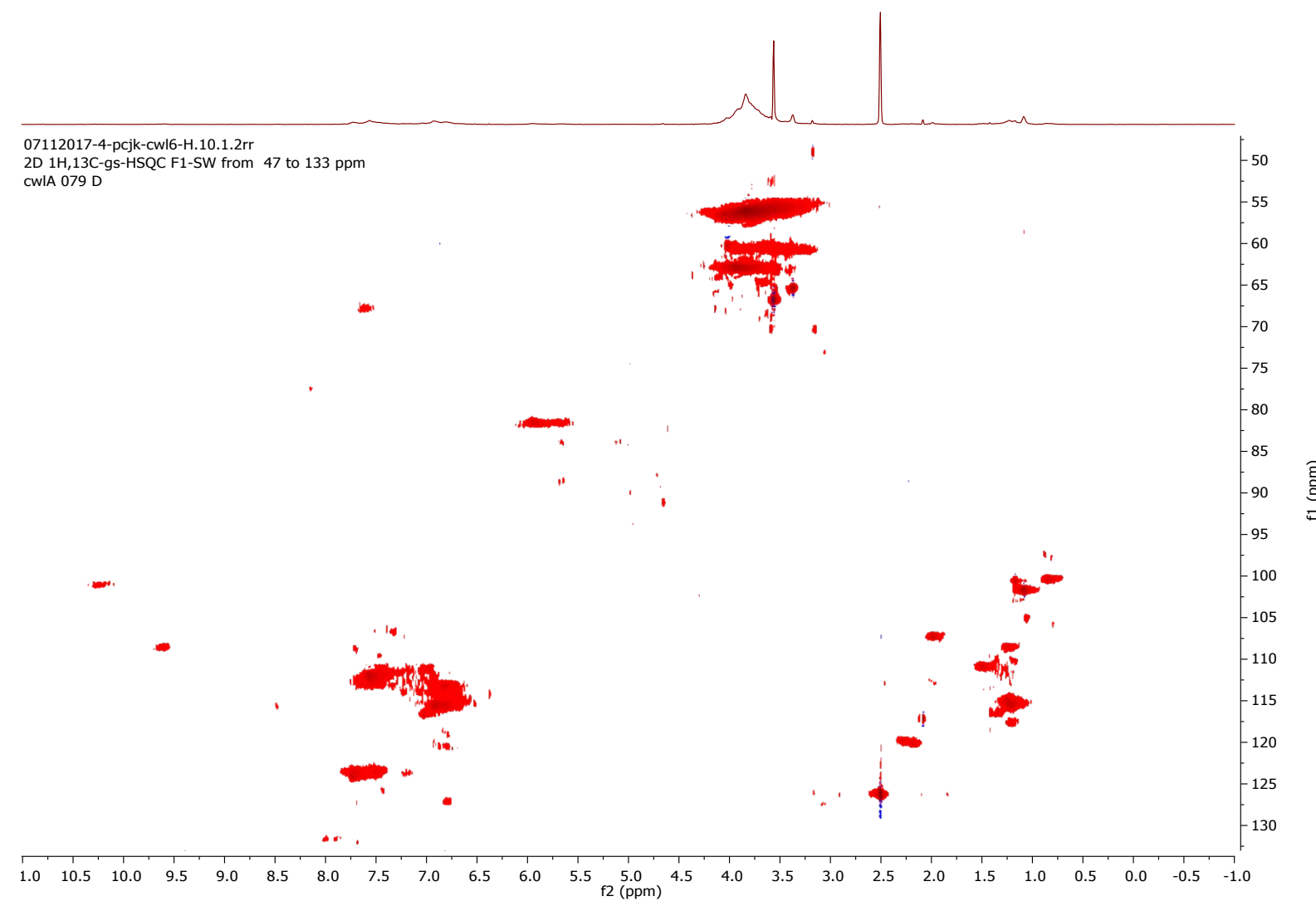

2D HSQC NMR of oxidised Douglas fir dioxosolv lignin in DMSO- $d_{6}$. Oxidised with 2 eq. DDQ at $60{ }^{\circ} \mathrm{C}$ for $24 \mathrm{hrs}$

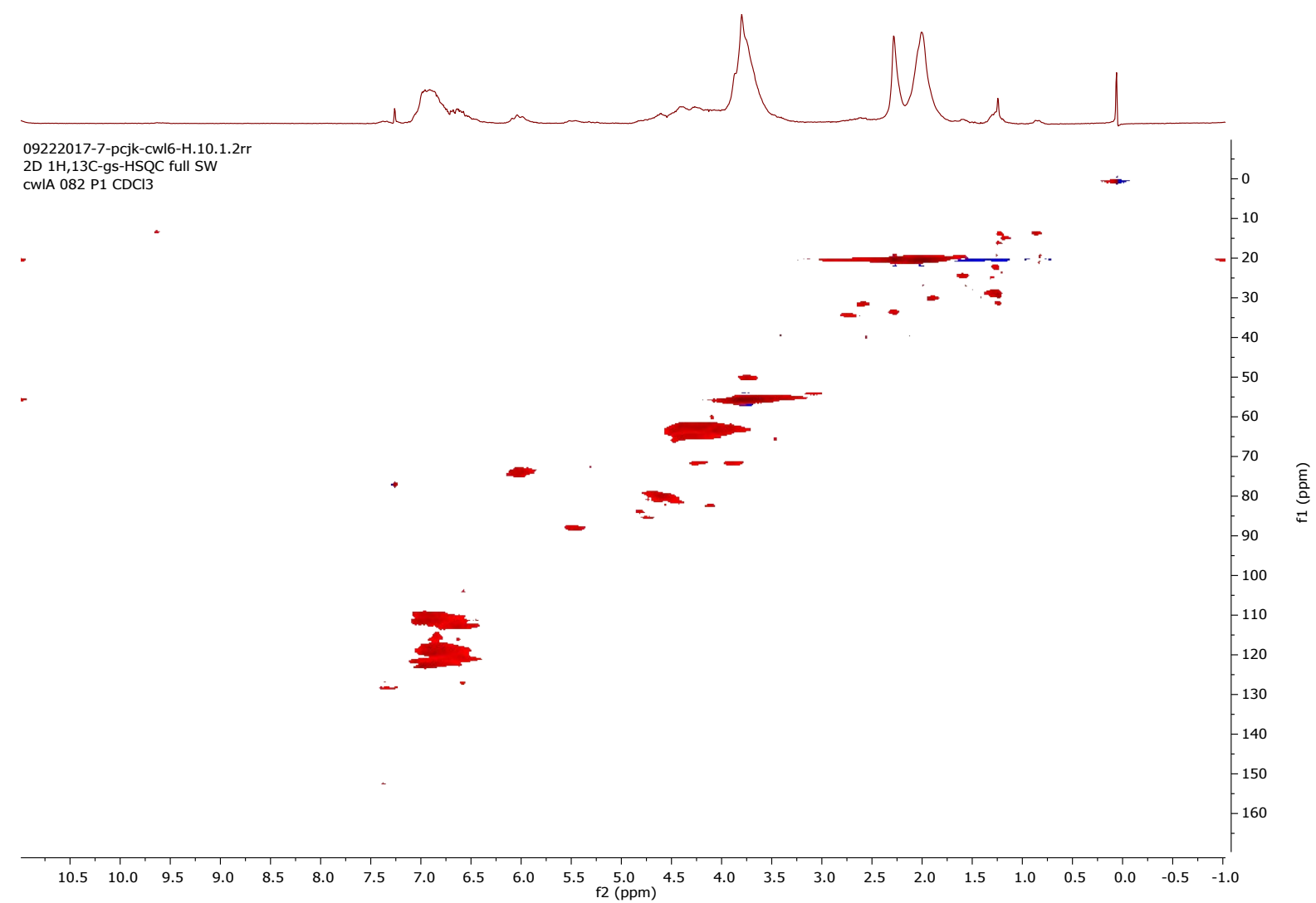

2D HSQC NMR of acetylated Douglas fir dioxosolv lignin in $\mathrm{CDCl}_{3}$. Acetylated following the procedure described above. 